Klas Diederich · John Erik Fornæss

\title{
Lineally convex domains of finite type: holomorphic support functions
}

Received: 11 November 2002 / Published online: 4 November 2003

\begin{abstract}
Smooth bounded lineally convex domains of finite type constitute a natural class of domains in complex analysis, since they are locally biholomorphically invariant. A smooth family of holomorphic support functions is constructed by an almost explicit formula on every such domain. It satisfies the best possible estimates near the point of support on every two-dimensional transverse affine intersection with the domain. Together with a suitable pseudometric on these domains, it will allow to do precise quantitative complex analysis by integral kernels on them.
\end{abstract}

\section{Introduction}

A very important tool for quantitative analysis on smoothly bounded pseudoconvex domains $D$ in $\mathbb{C}^{n}$ are smooth families of holomorphic support functions, i.e., smooth mappings $S: \partial D \times U \rightarrow \mathbb{C}$, which are holomorphic in the second variable on an open neighborhood $U$ of $\bar{D}$ and such that $S(\zeta, \zeta)=0$ and $\operatorname{Re} S \mid\{\zeta\} \times(\bar{D} \backslash\{\zeta\})<0$ for all $\zeta \in \partial D$. If they satisfy in addition optimal estimates (depending on the geometry of $\partial D$ ) near each $\zeta \in \partial D$, they allow to use the whole machinery of the so-called $\bar{\partial}$-solving Cauchy-Fantappié kernels to solve all kinds of quantitative problems of complex analysis in many different norms. Nice presentations of this theory are, for instance, given in the books by Henkin and Leiterer ([16] and [15]) and the recent book by I. Lieb and J. Michel ([20]).

In there article [3] M. Andersson and B. Berndtsson modified the HenkinRamirez formulas in such a way that they include certain weight factors and no derivatives of $S$ in the first variable. Both variations can be useful for the treatment of certain quantitative problems (see e.g. [6]), however it seems, that often kernels requiring higher regularity of $S$ in the first variable are needed (see e.g. [10]).

We want to remark here, that it is still a remote goal to treat the decisive quantitative problems of complex analysis, like the solution of the $\bar{\partial}$-equations in $L^{\infty}$-norms, via $L^{2}$-estimates. In any case this method applied in the very special cases where it can be made to work enforces a long detour which is unnecessary if

K. Diederich: Mathematik, Universität Wuppertal, Gausstr. 20, 42097 Wuppertal, Germany. e-mail: klas@math.uni-wuppertal.de

J.E. Fornæss: Department of Mathematics, University of Michigan, 2074 East Hall, Ann Arbor, MI 48109-1109, USA. e-mail: fornaess@ umich.edu

Mathematics Subject Classification (2000): 32F17, 32T25, 32T40 
smooth families of support functions can be directly constructed from the geometry of the domains.

The machinery of the Cauchy-Fantappié kernels reduces the quantitative analysis of smoothly bounded pseudoconvex domains in $\mathbb{C}^{n}$ essentially to two questions:

(a) the study of the local geometry at the boundary of the domains;

(b) the construction of smooth families of holomorphic support functions with optimal estimates.

The remaining work has in many cases become almost a routine with some additional considerations in each separate case (for recent work in this direction see for instance [6], [14], [12]).

However, unfortunately, not all 'decent' domains admit smooth families of holomorphic support functions in the above sense. For a study of this phenomenon in a series of examples, which have, in fact, all real-analytic boundaries of finite type, see [13]. Altogether, it is highly important to construct such families for as many classes of weakly pseudoconvex domains with smooth boundary as possible.

For strictly pseudoconvex domains this construction is standard (see for instance [16]). But for bounded pseudoconvex domains $D \subset \subset \mathbb{C}^{n}$ with smooth boundary the problem is in general much more delicate. It may still be relatively easy to construct a holomorphic support function near each fixed boundary point $\zeta \in \partial D$ satisfying optimal estimates. But since the rank of the Levi form restricted to the holomorphic tangent bundle and the direction of its eigenvectors might jump from point to point on the boundary, it is difficult to get families of holomorphic support functions with optimal estimates which depend in a $\mathcal{C}^{\infty}$ fashion on the boundary point where they are attached, if classes of domains are considered where such a jumping really can occur in a considerable manner.

Therefore, it was important to have an almost explicit construction of such families for bounded smoothly bounded convex domains of finite type as given by the authors in [7]. This construction became possible because of the so-called Bruna-Nagel-Wainger Lemma from [5] on values of convex functions.

The purpose of this article is to extend this construction to a considerably wider class of domains, namely the so-called bounded smoothly bounded weakly lineally convex domains of finite type (for simplicity we will drop in this article the word 'weakly' from this lengthy expression). This class of domains which comprises all bounded smoothly bounded convex domains of finite type, is considerably more general and also has the advantage of being invariant under biholomorphic transformations defined in a neighborhood of the closure of the respective domains. They have been originally introduced by Behnke and Peschl in 1935 in [2] where they were called 'planarkonvex'. They were extensively studied by Chr. Kiselman in [17], [18] and [19] and besides other related classes of domains also in [1]. In particular, [19] contains a characterization by a differential inequality, the so-called Behnke-Peschl condition, the necessity of which goes already back to [2] and will be important for us.

Because of the possible jumping of the rank of the Leviform and the eigenvectors of it for our domains, it is important to express the optimal estimates satisfied 
by $S$ in such a way that the point $\zeta \in \partial D$ where they are attached can move and such that they capture all directions in $T_{\zeta}^{10} \partial D$ well. We do this by stating these estimates on all 2-dimensional slices spanned by the normal to $\partial D$ at $\zeta$ and any direction in $T_{\zeta}^{10} \partial D$.

We can in many respects follow in our construction the scheme of [7] and will quite often refer to this paper. However, one of the new real difficulties here is, that lineally convex domains of course do not have convex defining functions such that we cannot directly make use of the important Bruna-Nagel-Wainger Lemma. However, we also cannot work without it. We, therefore, have to use our notion of "almost convexity" and have to show that certain perturbations of the Taylor polynomials arising from the defining functions of our domains are indeed convex (for details see Lemma 4.8). All this work was not necessary for bounded smooth convex domains of finite type. Because of the lack of convexity also the estimates of certain remainder terms in our construction has become considerably more complicated than in the convex case.

Once the $\mathcal{C}^{\infty}$ family of supporting functions with optimal estimates is available, there are many applications possible. We will explain this further in the last section. But we want to say already now, that an another important ingredient of such applications is a study of the local geometry adapted to the jumping rank of the Levi form and its eigenvectors. This has been extensively studied in the thesis of M. Conrad at the University of Wuppertal following ideas of E. Stein, A. Nagel and others for other classes of domains.

\section{Notations and results}

We consider in this article domains $D \subset \subset \mathbb{C}^{n}, 0 \in \partial D$, with the property, that there is an open neighborhood $W=W(0)$ such that $\partial D \cap W$ is $\mathcal{C}^{\infty}$-smooth and lineally convex in the sense that for all $z \in \partial D \cap W$ the intersection

$$
\left(z+T_{z}^{10} \partial D\right) \cap(D \cap W)=\varnothing
$$

We, furthermore, suppose, that $\partial D$ is of finite type at all points inside $W$. After shrinking $W$ we, then, may assume, that there is a $\mathcal{C}^{\infty}$ defining function $r: W \rightarrow \mathbb{R}$ for $D$ on $W$ and a number $\eta_{0}>0$ such that $\partial r(z) \neq 0$ for all $z \in W$ and all level sets $\partial D_{\eta}=\{z \in W: r(z)=\eta\}$ are lineally convex and of finite type for any $-\eta_{0} \leq \eta \leq \eta_{0}$. We put $W_{0}:=\left\{z \in W:|r(z)|<\eta_{0}\right\}$ and write $\partial D_{z}:=\left\{\zeta \in W_{0}:\right.$ $r(\zeta)=r(z)\}$ for any $z \in W_{0}$.

We observe at first, that the result of [4] does hold for lineally convex hypersurfaces of finite type. Hence the 1-type $\tau_{1}(z)$ of $\partial D_{\eta}$ at any point $z \in \partial D_{\eta}$, $-\eta_{0} \leq \eta \leq \eta_{0}$ is equal to the complex line-type. In particular, $\tau_{1}$ is upper semicontinuous on $W_{0}$. Therefore, we may assume, that $\tau_{1}$ attains its maximum, say $\hat{m}=2 m$ for some $m \in \mathbb{N}$, at 0 .

For any point $\zeta \in W_{0}$ we denote by $n_{\zeta}$ the exterior unit normal to $\partial D_{\zeta}$ at $\zeta$. Together with a unit vector $t \in T_{\zeta}^{10} \partial D_{\zeta} \backslash\{0\}$, we put

$$
A_{\zeta, t}:=\left\{z: z_{\zeta, t}(w):=\zeta-i w_{1} n_{\zeta}+w_{2} t \text { with } w=\left(w_{1}, w_{2}\right) \in \mathbb{C}^{2}\right\}
$$


and define

$$
\begin{gathered}
D_{\zeta, t}:=\left\{w \in \mathbb{C}^{2}: z_{\zeta, t}(w):=\zeta-i w_{1} n_{\zeta}+w_{2} t \in W_{0},\right. \\
\left.r_{\zeta, t}(w):=r\left(z_{\zeta, t}(w)\right)-r(\zeta)<0\right\}
\end{gathered}
$$

Notice, that $\partial D_{\zeta, t}$ is $\mathcal{C}^{\infty}$-smooth and lineally convex of type $\leq \hat{m}$ near 0 . In fact, we have

$$
D \cap A_{\zeta, t} \cap W_{0}=\left\{z_{\zeta, t}(w): w \in D_{\zeta, t}\right\}
$$

We put for $j=2, \ldots, 2 m$

$$
P_{\zeta, t}^{j}(w):=\sum_{k+l=j} \frac{1}{k !} \frac{1}{l !} \frac{\partial^{j} r_{\zeta, t}(0)}{\partial w_{2}^{k} \partial \bar{w}_{2}^{l}} w_{2}^{k} \bar{w}_{2}^{l}
$$

Notice, that the coefficients of $P_{\zeta, t}^{j}$ are $\mathcal{C}^{\infty}$ in $(\zeta, t)$.

In order to be able to formulate our main result, we need the following notation:

Definition 2.1. For any polynomial $\sum_{j=0}^{N} \sum_{|\alpha|+|\beta|=j} a_{\alpha \bar{\beta}} z^{\alpha} \bar{z}^{\beta}$ on any $\mathbb{C}^{k}$ we put

$$
\|P\|:=\sum_{j=0}^{N} \sum_{|\alpha|+|\beta|=j}\left|a_{\alpha \bar{\beta}}\right|
$$

With these notations and definitions our main result now can be stated as follows:

Theorem 2.2. For a suitable constant $\hat{c}>0$ and any $\varepsilon>0$ small enough there is a function $\hat{S}(z, \zeta) \in \mathcal{C}^{\infty}\left(\mathbb{C}^{n} \times W_{0}\right)$ which is a holomorphic polynomial of degree $2 m$ in $z$ for any fixed $\zeta \in W_{0}$, and there is a radius $d=d(\varepsilon)$, such that for $\zeta \in W_{0}$ one has $\hat{S}(\zeta, \zeta)=0$ and for each unit vector $t \in T_{\zeta}^{10} \partial D_{\zeta}$ the following estimate holds for the function $S_{\zeta, t}(w):=\hat{S}\left(z_{\zeta, t}(w), \zeta\right)$ as a function of $w$ on $|w|<d$ :

$$
\operatorname{Re} S_{\zeta, t}(w) \leq r_{\zeta, t}(w) h_{\zeta, t}(w)-\varepsilon \hat{c} \sum_{j=2}^{2 m}\left\|P_{\zeta, t}^{j}\right\|\left|w_{2}\right|^{j}
$$

where $h_{\zeta, t}(w)$ is a $\mathcal{C}^{\infty}$ function of $(\zeta, t, w)$ of the form $h_{\zeta, t}(w)=h\left(\zeta-i w_{1} n_{\zeta}+\right.$ $\left.w_{2} t, \zeta\right)$ with a positive $\mathcal{C}^{\infty}$ function $h$ on $\left\{z \in \mathbb{C}^{n}:|z|<d\right\} \times W_{0}$, bounded away from 0 .

Remark 2.3. The polynomial $P_{\zeta, t}^{j}$ also can be obtained in the following way: put

$$
P_{\zeta}^{j}(z):=\sum_{|\alpha|+|\beta|=j} \frac{1}{\alpha ! \beta !} \frac{\partial^{j} r(\zeta)}{\partial z^{\alpha} \partial \bar{z}^{\beta}}(z-\zeta)^{\alpha}(\bar{z}-\bar{\zeta})^{\beta}
$$

Then one has the equation

$$
P_{\zeta}^{j}\left(z_{\zeta, t}(w)\right)=P_{\zeta, t}^{j}(w)
$$


The estimate (2.6) for $\hat{S}$ has been formulated on 2-dimensional transverse slices, since it becomes more precise in this way. Depending on the respective purpose different estimates on $\mathbb{C}^{n}$ can be derived from this. We formulate here the following pointwise estimate, which, however, in general is much weaker than (2.6).

Corollary 2.4. Fix a point $\zeta \in W_{0}$ and choose coordinates $z$ (produced by an affine unitary cordinate change) centered at $\zeta$ which realize the multitype $\mathcal{M}=$ $\left(1, m_{2}, \ldots, m_{n}\right)$ of $\partial D_{\zeta}$ at $\zeta$. Then one has for a suitable constant $c>0$ in these coordinates the estimate

$$
\operatorname{Re} \hat{S}(z, 0) \leq c\left(r(z) h(z)-\sum_{j=2}^{n}\left|z_{j}\right|^{m_{j}}\right)
$$

for $|z|$ small enough.

Remark 2.5. It should be noticed, that the constant $c$ in the Corollary can, in general, not be chosen independently of $\zeta$.

The family of support functions $\hat{S}(z, \zeta)$ can be described quite explicitly. Namely, one has the following supplementary statement which will be proved together with Theorem 2.2:

Theorem 2.6. The $\mathcal{C}^{\infty}$ family of support functions $\hat{S}(z, \zeta)$ with the properties of Theorem 2.2 can be chosen in such a way, that the following holds true:

Let $M, K>0$ be chosen sufficiently large and $\varepsilon>0$ sufficiently small. Furthermore choose a $\mathcal{C}^{\infty}$ family $l_{\zeta}(\hat{z})$ of affine unitary transformations on $W_{0}$ translating $\zeta$ to 0 and rotating $n_{\zeta}$ to the vector $(i, 0, \ldots, 0) \in \mathbb{C}^{n}$. Then there is on $\zeta \in W_{0}$ a $\mathcal{C}^{\infty}$ family of holomorphic polynomials $A_{\zeta}, A_{\zeta}(0)=0$, such that the following holds:

If we introduce new coordinates $\tilde{z}=\left(\tilde{z}_{1}, \tilde{z}_{2}, \ldots, \tilde{z}_{n}\right)=\left(\tilde{z}_{1}, \tilde{z}^{\prime}\right)$ by putting $z=$ $l_{\zeta}(\hat{z})$ and then $\hat{z}=\Phi_{\zeta}(\tilde{z})$ with $\tilde{z}_{1}=\hat{z}_{1}\left(1-A_{\zeta}(\hat{z})\right)$ and $\tilde{z}_{k}=\hat{z}_{k}$ for $k=2, \ldots, n$, meaning that $\tilde{z}=\Phi_{\zeta}^{-1} \circ l_{\zeta}^{-1}(z)=: \tilde{\Phi}_{\zeta}^{-1}(z)$, then, for any $\zeta \in W_{0}$, the function $S_{\zeta}(\tilde{z}):=\hat{S}\left(\tilde{\Phi}_{\zeta}(\tilde{z}), \zeta\right)$ is given by the formula

$$
S_{\zeta}(\tilde{z})=\tilde{z}_{1}+K \tilde{z}_{1}^{2}-\varepsilon \sum_{j=2}^{2 m} M^{2^{j}} \sigma_{j} \sum_{\substack{|\alpha|=j \\ \alpha=\left(\alpha_{2}, \ldots, \alpha_{n}\right)}} \frac{1}{\alpha !} \frac{\partial^{j} \tilde{r}_{\zeta}(0)}{\partial \tilde{z}^{\alpha}} \tilde{z}^{\prime \alpha}
$$

where $\tilde{r}_{\zeta}(\tilde{z})=r\left(\tilde{\Phi}_{\zeta}(\tilde{z}), \zeta\right)-r(\zeta)$ and

$$
\sigma_{j}:=\left\{\begin{array}{ccc}
1 & \text { for } j \equiv 0 & \bmod 4 \\
-1 \text { for } j \equiv 2 & \bmod 4 \\
0 & \text { otherwise } &
\end{array}\right.
$$

In the new $\tilde{z}$-coordinates the estimate satisfied by $\operatorname{Re} S_{\zeta}(\tilde{z})$ and corresponding to (2.6) can be replaced by the following slightly stronger estimate: 


$$
\begin{aligned}
\operatorname{Re} S_{\zeta}\left(w_{1}(-i, 0, \ldots, 0)+w_{2} t\right) \leq & \tilde{r}_{\zeta, t}(\tilde{w}) \tilde{h}_{\zeta, t}(\tilde{w})-\frac{K}{2}\left(\operatorname{Re} \tilde{w}_{1}\right)^{2} \\
& -\varepsilon \tilde{c} \sum_{j=2}^{2 m}\left\|\tilde{P}_{\zeta, t}^{j}\right\|\left|\tilde{w}_{2}\right|^{j}
\end{aligned}
$$

where for any unit vector $t \in T_{\zeta}^{10} \partial D_{\zeta}$ we have put $\tilde{r}_{\zeta, t}(\tilde{w}):=\tilde{r}_{\zeta}\left(\tilde{w}_{1}(i, 0, \ldots, 0)+\right.$ $\left.\tilde{w}_{2} t\right)$ and where $\tilde{h}_{\zeta, t}(\tilde{w})$ is a $\mathcal{C}^{\infty}$ function of all its arguments of the form $\tilde{h}_{\zeta, t}(\tilde{w})=$ $\tilde{h}\left(\zeta, \zeta+\tilde{w}_{1}(-i, 0, \ldots, 0)+\tilde{w}_{2} t\right)$ with a function $\tilde{h}_{\zeta}(\tilde{z})$ which is $\mathcal{C}^{\infty}$ near $(0,0)$ in all its arguments and positive and bounded away from 0 . The polynomials $\tilde{P}_{\zeta, t}^{j}(\tilde{w})$, homogeneous of degree $j$, are defined to be $\tilde{P}_{\zeta, t}^{j}(\tilde{w}):=\sum_{k+l=j} \frac{1}{k ! l !} \frac{\partial^{j} \tilde{r}_{\zeta, t}(0)}{\partial \tilde{w}_{2}^{k} \partial \tilde{\tilde{w}}_{2}^{l}} \tilde{w}_{2}^{k} \overline{\tilde{w}}_{2}^{l}$.

Remark 2.7. It might be useful for the reader to observe already here, that the transformations $\tilde{z}=\tilde{\Phi}_{\zeta}^{-1}(z)$ map the affine spaces $A_{\zeta, t}$ from (2.1) into the spaces $A_{0, t}=\left\{\tilde{z}: \tilde{z}=w_{1}(-i, 0, \ldots, 0)+w_{2} t, w=\left(w_{1}, w_{2}\right) \in \mathbb{C}^{2}\right\}$.

In preparation of the following sections we still want to introduce some additional notations.

After using the coordinate transformations $l_{\zeta}(z)$ from Theorem 2.6 (but denoting also the new coordinates by $z$ ), we can write $\partial D_{\zeta}$ as a graph over the $\operatorname{Im} z_{1}=0$ hyperplane. This gives us defining functions of the form

$$
\begin{aligned}
r_{\zeta}^{g}(z) & =\operatorname{Im} z_{1}+\hat{R}_{\zeta}\left(\operatorname{Re} z_{1}, z^{\prime}\right) \\
& =\operatorname{Im} z_{1}+P_{\zeta}\left(z^{\prime}\right)+R_{\zeta}\left(\operatorname{Re} z_{1}, z^{\prime}\right)
\end{aligned}
$$

with $\hat{R}_{\zeta}(0)=0, d \hat{R}_{\zeta}(0)=0$ and $P_{\zeta}\left(z^{\prime}\right)$ consisting of all terms in $z^{\prime}$ only up to total order $2 m$ of the Taylor series of $\hat{R}_{\zeta}$.

We observe as in [7] that one has

$$
\left|R_{\zeta}\left(x_{1}, z^{\prime}\right)\right| \leq C\left(x_{1}^{2}+\left|x_{1}\right|\left|z^{\prime}\right|+\left|z^{\prime}\right|^{2 m+1}\right)
$$

for all $\left(x_{1}, z^{\prime}\right)$ close to 0 with a constant uniform in $\zeta \in W_{0}$. Furthermore, we have

$$
P_{\zeta}\left(z^{\prime}\right)=\sum_{j=2}^{2 m} P_{\zeta}^{j}\left(z^{\prime}\right)
$$

with

$$
\begin{aligned}
P_{\zeta}^{j}\left(z^{\prime}\right) & =\sum_{|\alpha|+|\beta|=j} \frac{1}{\alpha ! \beta !} \frac{\partial^{j} r_{\zeta}^{g}(0)}{\partial z^{\prime \alpha} \partial \bar{z}^{\prime \beta}} z^{\prime \alpha} \bar{z}^{\prime \beta} \\
& =\sum_{|\alpha|+|\beta|=j} a_{\alpha \bar{\beta}}(\zeta) z^{\prime \alpha} \bar{z}^{\prime \beta}
\end{aligned}
$$


Remark 2.8. 1) In the following we will most of the time work with the defining functions $r_{\zeta}^{g}$. We, therefore, will denote them again by $r_{\zeta}$, until the difference between the two will have to be considered at the end of the article. In particular, we also will use in (2.2) and (2.4) as $r_{\zeta, t}(w)$ the function

$$
r_{\zeta, t}(w):=r_{\zeta}^{g}\left(-w_{1}(i, 0, \ldots, 0)+w_{2} t\right)
$$

2) We point out, that $r_{\zeta}, r_{\zeta}^{g}, P_{\zeta}, P_{\zeta}^{j}$ are all $\mathcal{C}^{\infty}$ in $(z, \zeta)$.

Some parts of the proof of Theorems 2.2 and 2.6 carry over from the proof of Theorem 2.3 in [7]. We will not repeat them in the following. However, we will always make sure, that the reader can follow the proof of the Theorems in this article directly, if he is willing to accept that certain Lemmas stated explicitly here have been established in [7]. The important difference to [7] consists, of course, in the fact, that we cannot use convexity of the defining functions here, such that also the Bruna-Nagel-Wainger Lemma from [5], which was an extremely important tool in [7], can no longer be used directly. We will, however, prove certain structure Lemmas for defining functions of lineally convex domains which will allow us to still apply this Lemma in a very useful way after suitable modifications (see for instance Lemma 4.9 below).

\section{Some estimates for lineally convex domains}

We will need some basic estimates involving the real Hessian and the Leviform of lineally convex domains. And since our construction of support functions will be done for the domains $D_{\zeta, t} \subset \mathbb{C}^{2}$ as defined in (2.2) (see also (2.3)), we will prove these estimates here only for (locally near 0 ) lineally convex domains in $\mathbb{C}^{2}$. For this purpose we let $D$ be one of the domains $D_{\zeta, t}$ from (2.2) and denote from now on the coordinates $\left(w_{1}, w_{2}\right)$ as introduced in $(2.1)$ by $(w, z) \subset \mathbb{C}^{2}$ splitting $w$ as $w=u+i v$.

Let now $r$ be an arbitrary defining function of $D$ near 0 and let $p \in \partial D$ be an arbitrary point (close enough to 0 ). Then the complex tangent line to $\partial D$ at $p$ is parametrized by

$$
\mathbb{C} \ni \tau \longmapsto p+\tau \cdot\left(r_{w}(p),-r_{z}(p)\right) \in \mathbb{C}^{2}
$$

Hence, the condition of lineal convexity of $D$ at $p$ means that

$$
g(\tau):=r\left(p+\tau\left(r_{w}(p),-r_{z}(p)\right)\right) \geq 0
$$

Set $A:=g_{\tau \bar{\tau}}(p)$ and $B:=\frac{g_{\tau \tau}(p)}{2}$. Since the second order part of the Taylor series of $g$ at 0 is

$$
A|\tau|^{2}+B \tau^{2}+\overline{B \tau}^{2} \geq 0
$$

we get from (3.1) as condition for lineal convexity the inequality $|B| \leq \frac{A}{2}$. After a small calculation this proves the following 
Lemma 3.1. Let $p \in \partial D$ be arbitrary (close to 0 ) and set

$$
A:=r_{z \bar{z}}\left|r_{w}\right|^{2}-r_{z} r_{z} r_{\bar{w}}-r_{\bar{z} w} r_{z} r_{\bar{w}}+r_{w \bar{w}}\left|r_{z}\right|^{2}
$$

and

$$
2 B:=r_{z z} r_{w}^{2}-r_{z w} r_{w} r_{z}-r_{z w} r_{z} r_{w}+r_{w w} r_{z}^{2}
$$

(all derivatives of $r$ are taken at $p$ ). Then $2|B| \leq A$.

Remark 3.2. This Lemma gives the necessity of the so-called Behnke-Peschl condition in the case of $\mathbb{C}^{2}$. It has already been shown in $\mathbb{C}^{n}$ in [2]. Its sufficiency for the characterization of smooth lineally convex domains has been proved in [19]. Our formulation (and proof) are adapted to our needs.

We now assume, that the domain $D$ is of finite type $\hat{m}=2 m$ and use the same coordinates $(w, z) \in \mathbb{C}^{2}$ as in (2.13) such that a $D$ has near 0 a defining function of the form $r=v+R(u, z)$, in detail

$$
r(w, z)=v+a u^{2}+u \sum_{j=1}^{m} Q^{j}(z)+\sum_{l=2}^{2 m} P^{l}(z)+O\left(u^{3}, u^{2} z, u z^{m+1}, z^{2 m+1}\right)
$$

We now want to make Lemma 3.1 more explicit by calculating the expressions (3.2) and (3.3) in terms of (3.4). We get at first the following expressions for the needed derivatives of $r$ :

$$
\begin{aligned}
r_{z} & =u \sum_{j=1}^{m} Q_{z}^{j}+\sum_{l=2}^{2 m} P_{z}^{l}+O\left(u^{2}, u z^{m}, z^{2 m}\right) \\
r_{w} & =\frac{1}{2 i}+a u+\frac{1}{2} \sum_{j=1}^{m} Q^{j}+O\left(u^{2}, u z, z^{m+1}\right) \\
r_{z z} & =u \sum_{j=1}^{m} Q_{z z}^{j}+\sum_{l=2}^{2 m} P_{z z}^{l}+O\left(u^{2}, u z^{m-1}, z^{2 m-1}\right) \\
r_{z \bar{z}} & =u \sum_{j=1}^{m} Q_{z \bar{z}}^{j}+\sum_{l=2}^{2 m} P_{z \bar{z}}^{l}+O\left(u^{2}, u z^{m-1}, z^{2 m-1}\right) \\
r_{z w} & =\frac{1}{2} \sum_{j=1}^{m} Q_{z}^{j}+O\left(u, z^{m}\right) \\
r_{z \bar{w}} & =\frac{1}{2} \sum_{j=1}^{m} Q_{z}^{j}+O\left(u, z^{m}\right) \\
r_{w w} & =\frac{a}{2}+O(u, z) \\
r_{w \bar{w}} & =\frac{a}{2}+O(u, z)
\end{aligned}
$$


Using these expressions a straightforward although somewhat tedious calculation, which we skip here, gives

$$
\begin{aligned}
r_{z \bar{z}}\left|r_{w}\right|^{2}= & \frac{u}{4} \sum_{j=1}^{m} Q_{z \bar{z}}^{j}+\frac{u}{4}\left(\sum_{j=1}^{m} Q_{z \bar{z}}^{j}\right)\left(\sum_{j=1}^{m} Q_{j}\right)^{2} \\
& +O\left(u z, z^{m+1}\right) \sum_{l=2}^{2 m} P_{z \bar{z}}^{l}+\frac{1}{4} \sum_{l=2}^{2 m} P_{z \bar{z}}^{l}+\frac{1}{4}\left(\sum_{l=2}^{2 m} P_{z \bar{z}}^{l}\right)\left(\sum_{j=1}^{m} Q_{j}\right)^{2} \\
& +O\left(u^{2}, u z^{m-1}, z^{2 m-1}\right)
\end{aligned}
$$

In the same way we get

$$
\begin{aligned}
r_{w \bar{w}}\left|r_{z}\right|^{2}= & O(u) \cdot \sum_{l=2}^{2 m} P_{\bar{z}}^{l}+\left(\frac{a}{2}+O(z)\right)\left|\sum_{l=2}^{2 m} P_{z}^{l}\right|^{2} \\
& +O\left(u^{2}, u z^{m-1}, z^{2 m-1}\right)
\end{aligned}
$$

and

$$
\begin{aligned}
r_{z} r_{w} r_{\bar{z}}= & O\left(u, z^{m}\right) \cdot\left(\sum_{l=2}^{2 m} P_{\bar{z}}^{l}\right)+\frac{u}{4 i}\left|\sum_{j=1}^{m} Q_{z}^{j}\right|^{2}+\frac{u}{4}\left|\sum_{j=1}^{m} Q_{z}^{j}\right|^{2} \sum_{j=1}^{m} Q^{j} \\
& +\frac{1}{2} \sum_{j=1}^{m} Q_{z}^{j} \cdot\left(\frac{1}{2 i}+\frac{1}{2} \sum_{j=1}^{m} Q_{j}\right) \cdot\left(\sum_{l=2}^{2 m} P_{\bar{z}}^{l}\right) \\
& +O\left(u^{2}, u z^{m-1}, z^{2 m-1}\right)
\end{aligned}
$$

and

$$
\begin{aligned}
r_{z z} r_{w}^{2}= & -\frac{u}{4} \sum_{j=1}^{m} Q_{z z}^{j}+\frac{u}{4}\left(\sum_{j=1}^{m} Q_{z z}^{j}\right)\left(\sum_{j=1}^{m} Q^{j}\right)^{2} \\
& +u\left(\sum_{j=1}^{m} Q_{z z}^{j}\right) \cdot \frac{1}{2 i}\left(\sum_{j=1}^{m} Q_{j}\right) \\
& +O\left(u z, z^{m+1}\right) \sum_{l=2}^{2 m} P_{z z}^{l}-\frac{1}{4}\left(\sum_{l=2}^{2 m} P_{z z}^{l}\right)+\frac{1}{4}\left(\sum_{l=2}^{2 m} P_{z z}^{l}\right)\left(\sum_{j=1}^{m} Q_{j}\right)^{2} \\
& +\frac{a u}{i} \sum_{l=2}^{2 m} P_{z z}^{l}+\frac{1}{2 i} \sum_{l=2}^{2 m} P_{z z}^{l} \sum_{l=2}^{m} Q_{j}+O\left(u^{2}, u z^{m-1}, z^{2 m-1}\right)
\end{aligned}
$$

as well as 


$$
\begin{aligned}
r_{z w} r_{w} r_{z}= & O\left(u, z^{m}\right) \cdot\left(\sum_{l=2}^{2 m} P_{z}^{l}\right)+\frac{u}{4 i}\left(\sum_{j=1}^{m} Q_{z}^{j}\right)^{2}+\frac{u}{4}\left(\sum_{j=1}^{m} Q_{z}^{j}\right)^{2}\left(\sum_{j=1}^{m} Q^{j}\right) \\
& +\frac{1}{4 i}\left(\sum_{j=1}^{m} Q_{z}^{j}\right)\left(\sum_{l=2}^{2 m} P_{z}^{l}\right)+\frac{1}{4}\left(\sum_{j=1}^{m} Q_{z}^{j}\right)\left(\sum_{j=1}^{m} Q^{j}\right)\left(\sum_{l=2}^{2 m} P_{z}^{l}\right) \\
& +O\left(u^{2}, u z^{m-1}, z^{2 m-1}\right)
\end{aligned}
$$

and finally

$$
\begin{aligned}
r_{w w}\left(r_{z}\right)^{2}= & a u\left(\sum_{j=1}^{m} Q_{z}^{j}\right)\left(\sum_{l=2}^{2 m} P_{z}^{l}\right) \\
& +\frac{a}{2}\left(\sum_{l=2}^{2 m} P_{z}^{l}\right)^{2}+O(z u)\left(\sum_{j=1}^{m} Q_{z}^{j}\right)\left(\sum_{l=2}^{2 m} P_{z}^{l}\right) \\
& +O(z, u)\left(\sum_{l=2}^{2 m} P_{z}^{l}\right)^{2}+O\left(u^{2}, u z^{m-1}, z^{2 m-1}\right)
\end{aligned}
$$

By putting all this into formula (3.2) one obtains after some lengthy calculation the following important estimates for $A$ :

$$
\begin{aligned}
& A=O\left(u, z^{m}\right) \cdot\left(\sum_{l=2}^{2 m} P_{z}^{l}\right)+O(z)\left|\sum_{l=2}^{2 m} P_{z}^{l}\right|^{2} \\
& +O\left(u z, z^{m+1}\right) \sum_{l=2}^{2 m} P_{z \bar{z}}^{l}+\frac{1}{4} \sum_{l=2}^{2 m} P_{z \bar{z}}^{l} \\
& +\frac{u}{4} \sum_{j=1}^{m} Q_{z \bar{z}}^{j}+\frac{u}{4}\left(\sum_{j=1}^{m} Q_{z \bar{z}}^{j}\right)\left(\sum_{j=1}^{m} Q^{j}\right)^{2} \\
& +\frac{1}{4}\left(\sum_{l=2}^{2 m} P_{z \bar{z}}^{l}\right)\left(\sum_{j=1}^{m} Q^{j}\right)^{2} \\
& -\frac{u}{2}\left|\sum_{j=1}^{m} Q_{z}^{j}\right|^{2} \sum_{j=1}^{m} Q^{j}+\frac{1}{2}\left(\sum_{j=1}^{m} Q_{\bar{z}}^{j}\right)\left(\frac{1}{2 i}-\frac{1}{2} \sum_{j=2}^{m} Q^{j}\right)\left(\sum_{l=2}^{2 m} P_{z}^{l}\right) \\
& -\frac{1}{2}\left(\sum_{j=1}^{m} Q_{z}^{j}\right)\left(\frac{1}{2 i}+\frac{1}{2} \sum_{j=1}^{m} Q^{j}\right)\left(\sum_{l=2}^{2 m} P_{\bar{z}}^{l}\right) \\
& +\frac{a}{2}\left|\sum_{l=2}^{2 m} P_{z}^{l}\right|^{2}+O\left(u^{2}, u z^{m-1}, z^{2 m-1}\right)
\end{aligned}
$$


and for $2 B$

$$
\begin{aligned}
2 B= & O\left(u, z^{m}\right)\left(\sum_{l=2}^{2 m} P_{z}^{l}\right)+O(z)\left(\sum_{l=2}^{2 m} P_{z}^{l}\right)^{2}+O\left(u z, z^{m+1}\right) \sum_{l=2}^{2 m} P_{z z}^{l}-\frac{1}{4} \sum_{L 02}^{2 m} P_{z z}^{l} \\
& -\frac{u}{4} \sum_{j=1}^{m} Q_{z z}^{j}+\frac{u}{4}\left(\sum_{j=1}^{m} Q_{z z}^{j}\right)\left(\sum_{j=1}^{m} Q^{j}\right)^{2}+\frac{1}{4}\left(\sum_{l=2}^{2 m} P_{z z}^{l}\right)\left(\sum_{j=1}^{m} Q^{j}\right)^{2} \\
& -\frac{u}{2}\left(\sum_{j=1}^{m} Q_{z}^{j}\right)^{2}\left(\sum_{j=1}^{m} Q^{j}\right)-\left(\sum_{j=1}^{m} Q_{z}^{j}\right)\left(\frac{1}{2 i}+\frac{1}{2} \sum_{j=1}^{m} Q^{j}\right) \sum_{l=2}^{2 m} P_{z}^{l} \\
& +\frac{a}{2}\left(\sum_{l=2}^{2 m} P_{z}^{l}\right)^{2}+\frac{a}{i} u \sum_{l=2}^{2 m} P_{z z}^{l}+\frac{u}{2 i}\left(\sum_{j=1}^{m} Q_{z z}^{j}\right)\left(\sum_{j=1}^{m} Q^{j}\right) \\
& +\frac{1}{2 i} \sum_{l=2}^{2 m} P_{z z}^{l} \sum_{j=1}^{m} Q^{j}-\frac{1}{2 i} u\left(\sum_{j=1}^{m} Q_{z}^{j}\right)^{2}+O\left(u^{2}, u z^{m-1}, z^{2 m-1}\right)
\end{aligned}
$$

The formulas (3.12) and (3.13) will be the main tools for our further investigation of the properties of the defining functions of lineally convex domains of finite type in $\mathbb{C}^{2}$.

\section{Basic properties of defining functions}

Let $D \subset \subset \mathbb{C}^{2}$ be a domain whith a $\mathcal{C}^{\infty}$-smooth boundary of finite type $\leq 2 m$ near $0 \in \partial D$. Suppose, the coordinates $(w, z)$ on $\mathbb{C}^{2}$ and a defining function $r$ of $D$ near 0 have been chosen such that (3.4) holds. Then the following holds

Lemma 4.1. Let $P^{j_{0}}$ be the lowest order non-vanishing term among all the $P^{j}$ from (3.4). Then $P^{j_{0}}$ is convex.

Before we give the proof we remind the reader of the following Lemma 3.4 of [7]

Lemma 4.2. A real-valued $\mathcal{C}^{2}$-function $\lambda$ on an open set $U \subset \mathbb{C}$ is convex on $U$ if and only if

$$
\Delta_{c} \lambda(z):=\frac{\partial^{2} \lambda}{\partial z \partial \bar{z}}(z)-\left|\frac{\partial^{2} \lambda(z)}{\partial z^{2}}\right| \geq 0 \quad \forall z \in U
$$

Proof of Lemma 4.1. From (3.12) and (3.13) we get for $u=0$

$$
\begin{aligned}
A & =\frac{1}{4} P_{z \bar{z}}^{j_{0}}+O\left(z^{j-1}\right) \\
2 B & =-\frac{1}{4} P_{z z}^{j_{0}}+O\left(z^{j-1}\right)
\end{aligned}
$$

Hence one has because of Lemma 3.1

$$
\left|P_{z z}^{j_{0}}\right| \leq P_{z \bar{z}}^{j_{0}}
$$

Together with Lemma 4.2 this gives the convexity of $P^{j_{0}}$. 
We now come back to the notations introduced in section 2, in particular, in (2.13) to (2.17). We denote the coordinates by $(w, z)$ instead of $\left(w_{1}, w_{2}\right)$ and write $\mathcal{R}$ for the family of defining functions $r_{\zeta, t}(w, z)$ appearing in (2.17). We put $m:=\frac{\hat{m}}{2}$ and denote by $\mathcal{F}$ the family of all polynomials $P_{\zeta, t}(z)$ of degree $\leq 2 m$ appearing when the decomposition (2.13) is written down for the functions from $\mathcal{R}$. Any $P \in \mathcal{F}$ is decomposed in the form

$$
P(z)=\sum_{j=2}^{2 m} P^{j}(z)
$$

with $P^{j}$ homogeneous of order $j$. Notice that $r_{0}:=r_{0,(1,0, \ldots, 0)}^{g} \in \mathcal{R}$ and that $P_{0}(z):=P_{0,(1,0, \ldots, 0)}(z) \in \mathcal{F}$. The families $\mathcal{R}$ and $\mathcal{F}$ are open neighborhoods of $r_{0}$ and $P_{0}$ respectively, which will have to be shrunk several times in the following. Notice also, that, according to an observation from the beginning of section 2 , the type of the domain $D_{\zeta,(1,0, \ldots, 0)}$ may be assumed to be $2 k \leq 2 m$. Hence $P_{0}^{j}=0$ for all $j=2, \ldots, 2 k-1$ and $P_{0}^{2 k} \neq 0$.

Our next goal is to carry over Lemma 4.2 from [7] to our new family $\mathcal{F}$ as defined here. The difficulty in doing so is, of course, the possible failure of convexity for the polynomials in $\mathcal{F}$.

For radii $0 \leq R^{\prime} \leq R^{\prime \prime}$ we put $A_{R^{\prime}, R^{\prime \prime}}:=\left\{z \in \mathbb{C}: R^{\prime} \leq|z| \leq R^{\prime \prime}\right\}$. Let $\hat{R}>0$ be arbitrarily small. Then, after shrinking $\mathcal{F}$ (and $\mathcal{R}$ accordingly), we can conclude, that for any $P \in \mathcal{F}$ there is a radius $0 \leq R_{2 k-1}<R_{2 k}=: \hat{R}$ such that

$$
\left\|P_{2 k}\right\||z|^{2 k} \geq \max _{j \neq 2 k}\left\|P^{j}\right\||z|^{j} \quad \forall z \in A_{R_{2 k-1}, R_{2 k}}
$$

Continuing this argument we get by the same reasoning as before Lemma 4.1 from [7]

Lemma 4.3. For any $P \in \mathcal{F}$ there are radii $0=R_{1} \leq \cdots \leq R_{2 k}=\cdots=R_{2 m}$ such that for all $2 \leq l \leq 2 m$ and all $z \in A_{l}:=A_{R_{l-1}, R_{l}}$ the estimate

$$
\left\|P^{l}\right\||z|^{l}>\max _{j \neq l}\left\|P^{j}\right\||z|^{j}
$$

holds.

It is crucial for the goal of this article that despite of the lack of convexity the following analogue of Lemma 4.2 from [7] still can be proved

Lemma 4.4. For any number $\delta>0$ there is after shrinking $\mathcal{F}$ (and $\mathcal{R}$ accordingly) to a sufficiently small neighborhood of $P_{0}$, a number $L_{\delta}>1$, such that for any $P \in \mathcal{F}$ and any $k, 2 \leq k \leq 2 m$, for which the radii $R_{k-1}, R_{k}$ chosen for $P$ as in Lemma 4.3 satisfy $\frac{R_{k}}{R_{k-1}} \geq \bar{L}_{\delta}$ one has

$$
\Delta_{c}\left(P^{k}\right)(z) \geq-\delta\left\|P^{k}\right\||z|^{k-2} \quad \forall z
$$


Proof. Notice at first that because of Lemma 4.2 for any $P \in \mathcal{F}$ the lowest order term $P^{k}$ (meaning that $P^{j}=0$ for all $2 \leq j<k$ ) is convex. Hence, in this case, we even have $\Delta_{c}\left(P^{k}\right)(z) \geq 0 \forall z$.

Next we observe that for $u=0$ the estimates (3.12) and (3.13) take the form

$$
\begin{aligned}
A & =\frac{1}{4} \sum_{l=2}^{2 m} P_{z \bar{z}}^{l}+O\left(\sum_{l=2}^{2 m}\left\|P^{l}\right\||z|^{l-1}\right)+O\left(z^{2 m-1}\right) \\
2 B & =-\frac{1}{4} \sum_{l=2}^{2 m} P_{z z}^{l}+O\left(\sum_{l=2}^{2 m}\left\|P^{l}\right\||z|^{l-1}\right)+O\left(z^{2 m-1}\right)
\end{aligned}
$$

From this we get using Lemma 3.1

$$
\begin{aligned}
\Delta_{c}\left(P^{k}\right)= & P_{z \bar{z}}^{k}-\left|P_{z z}^{k}\right| \\
\geq & 4\left[A-C \sum_{l=2}^{2 m}\left\|P^{l}\right\||z|^{l-1}-\frac{1}{4} \sum_{l \neq k} P_{z \bar{z}}^{l}\right] \\
& -4\left|-2 B+\frac{1}{4} \sum_{l \neq k} P_{z z}^{l}\right|-C \sum_{l}\left\|P^{l}\right\||z|^{l-1} \\
\geq & 4(A-2|B|)-\hat{C} \sum_{l}\left\|P^{l}\right\||z|^{l-1}-\hat{C} \sum_{l \neq k}\left\|P^{l}\right\||z|^{l-2} \\
\geq & -\tilde{C} \sum_{l \neq k}\left\|P^{l}\right\||z|^{l-2}-\tilde{C}\left\|P^{k}\right\||z|^{k-1}
\end{aligned}
$$

We define for any $2 \leq k \leq 2 m$ with $R_{k-1} \neq 0$

$$
\hat{R}_{k}:=\sqrt{R_{k-1} R_{k}}
$$

and put for fixed such $k$ the quotient $\frac{R_{k}}{R_{k-1}}=: L$. Then we choose $l \neq k$ and claim

$$
\left\|P^{l}\right\|\left(\hat{R}_{k}\right)^{l-2} \leq\left\|P^{k}\right\|\left(\hat{R}_{k}\right)^{k-2} \frac{1}{L^{\frac{|k-l|}{2}}}
$$

For the case $l>k$ the proof of this claim is as in [7] and for $l<k$ the same proof works as for $l>k$.

Putting (4.4) and (4.5) together and taking into account that $L \geq L_{\delta}$, we have now proved for $|z|=\hat{R}_{k}$

$$
\Delta_{c} P_{k} \geq-C\left\|P_{k}\right\| \hat{R}^{k-2}\left(\sum_{l \neq k} \frac{1}{\frac{|l-k|}{2}}+\hat{R}_{k}\right)
$$

If we suppose, that $L_{\delta}$ is chosen large enough and if $\hat{R}_{k}$ small enough (this, of course, means, that $\mathcal{R}$ and $\mathcal{F}$ have to be shrunk), the Lemma follows. 
We remind the reader of the following fact, which has been proved as Lemma 3.5 in [7]

Lemma 4.5. For every $j, 2 \leq j \leq 2 m$ there is a number $\delta_{j}>0$, such that the following holds: If $P^{j}(z)=\sum_{l+k=j} a_{l \bar{k}} z^{l} \bar{z}^{k}$ is a real-valued polynomial on $\mathbb{C}$ which is homogeneous of degree $j$ and which is almost convex in the sense that there is a $0<\delta<\delta_{j}$ such that

$$
\Delta_{c} P^{j} \geq-\delta\left\|P^{j}\right\||z|^{j-2}
$$

then the inequality

$$
\sigma_{j} \operatorname{Re}\left(a_{j 0} z^{j}\right) \geq \delta\left\|P^{j}\right\||z|^{j}
$$

holds for all $z \in \mathbb{C}$ for which

$$
P^{j}(z) \leq \delta\left\|P^{j}\right\||z|^{j}
$$

An immediate consequence of this Lemma is the following estimate

Lemma 4.6. Let $P^{j}, \delta$ be as in the previous Lemma. Then one has for $\varepsilon>0$ small enough

$$
\left|P^{j}(z)\right|+\varepsilon \operatorname{Re}\left(\sigma_{j} a_{j 0} z^{j}\right) \geq \varepsilon \delta\left\|P^{j}\right\||z|^{j} \quad \forall z
$$

Proof. For those points $z$ for which (4.7) holds, this follows directly from (4.6). If, on the other hand, $z$ is such that $P^{j}(z)>\delta\left\|P^{j}\right\||z|^{j}$, then (4.8) also follows if $\varepsilon>0$ is small enough.

Since in Lemma 4.4 we only consider the degrees $k$, for which $\frac{R_{k}}{R_{k-1}}$ is "large", although in the final estimations all terms $P^{k}$ have to be taken into account, we will have to use Lemma 4.3 from [7] without change. For the convenience of the reader we state it here.

Lemma 4.7. Let $\delta>0$ be fixed and choose $L_{\delta}>0$ and the neighborhood $\mathcal{F}$ as in Lemma 4.4. Also fix a constant $\hat{C}>0$. Then for any sufficiently large constant $M>0$ the following holds true:

Suppose the pair $(j, k), 2<j<k \leq 2 r$, has the following properties with respect to a $P \in \mathcal{F}$ :

a) $\frac{R_{l}}{R_{l-1}} \leq L_{\delta}$ for all $j<l<k$,

b) $\stackrel{\circ}{A}_{j} \neq \emptyset$ and $\AA_{k} \neq \emptyset$.

Then one has

$$
M^{2^{k}}\left\|P^{k}\right\||z|^{k}+M^{2^{j}}\left\|P^{j}\right\||z|^{j}>\hat{C} M^{2^{l}}\left\|P^{l}\right\||z|^{l} \quad \forall j<l<k, \quad \forall z
$$

In contrast to the situation of convex domains of finite type our functions $r \in \mathcal{R}$ are in general not convex. Hence, we cannot use Lemma 4.4 from [7] in our situation. We replace it by the following slight variation. 
Lemma 4.8. For all $\eta>0$ there is a constant $A_{\eta}>0$ such that for any $P \in \mathcal{F}$ the polynomial

$$
\rho_{\eta}:=\sum_{j=2}^{2 m} P^{j}(z)+\eta \sum_{j=2}^{2 m}\left\|P^{j}\right\||z|^{j}+A_{\eta} \cdot|z|^{2 m+1}
$$

is convex.

Proof. From the definition of $\rho_{\eta}$ one obtains

$$
\rho_{\eta, z \bar{z}}=\sum_{j=2}^{2 m} P_{z \bar{z}}^{j}+\eta \sum_{j=2}^{2 m} \frac{j^{2}}{4}\left\|P^{j}\right\||z|^{j-2}+\frac{(2 m+1)^{2}}{4} A_{\eta}|z|^{2 m-1}
$$

and

$$
\rho_{\eta, z z}=\sum_{j=2}^{2 m} P_{z z}
$$

Hence together with 4.3 we get

$$
\begin{aligned}
\frac{1}{4} \rho_{\eta, z \bar{z}}-\frac{1}{4}\left|\rho_{\eta, z z}\right| \geq & A(z, 0)-C \sum_{l=2}^{2 m}\left\|P^{l}\right\||z|^{l-1}+\left(\frac{(2 m+1)^{2}}{4} A_{\eta}-C\right)|z|^{2 m-1} \\
& +\frac{\eta}{4} \sum_{j=2}^{2 m} \frac{j^{2}}{4}\left\|P^{j}\right\||z|^{j-2}-2|B| \\
\geq & 0
\end{aligned}
$$

Because of Lemma 4.2 this means, that $\rho_{\eta}$ is convex.

Applying the so-called Bruna-Nagel-Wainger-Lemma, Lemma 2.1 from [5], we get

Lemma 4.9. There exists a universal constant $C_{m}>0$, such that for any $\eta>0$ there is a constant $A_{\eta}>0$, such that one has for all $P \in \mathcal{F}$ the associated polynomial $\rho_{\eta}$ from (4.9) satisfies the inequality

$$
\rho_{\eta}(z) \geq C_{m}\left(\left.\left.\sum_{j=2}^{2 m}\left|P^{j}(z)+\eta\left\|P^{j}\right\|\right| z\right|^{j}\left|+A_{\eta} \cdot\right| z\right|^{2 m+1}\right) \quad \forall z
$$

In the next Lemma much of the previous information is put together (and $\sigma_{j}$ is as in (2.11)). For simplicity of notation we call for any given $P \in \mathcal{F}$ an index $j$, $2 \leq j<2 m$, good, if for a given $\delta>0$ and $L_{\delta}$ chosen according to Lemma 4.4 the corresponding annulus $A_{j}$ satisfies $\frac{R_{j}}{R_{j-1}}>L_{\delta}$. Otherwise, $j$ obviously is bad.

Lemma 4.10. Choose $\mathcal{F}, \delta, L_{\delta}, M, \hat{C}$ as above and let $C_{m}$ be the constant from Lemma 4.9. Then for all $P \in \mathcal{F}$, any $\varepsilon>0$ and any pair $\left(\eta, A_{\eta}\right)$ as in Lemma 4.8 we have $\forall z$ the estimate

$$
\begin{aligned}
& \sum_{j=2}^{2 m} P^{j}+\eta \sum_{j=2}^{2 m}\left\|P^{j}\right\||z|^{j}+A_{\eta}|z|^{2 m+1}+\varepsilon \sum_{j=2}^{2 m} M^{2^{j}} \sigma_{j} \operatorname{Re}\left(a_{j 0} z^{j}\right) \\
& \geq C_{m}\left(\left.\left.\sum_{j \text { good }}\left|P^{j}+\eta\left\|P^{j}\right\|\right| z\right|^{j}\left|+A_{\eta}\right| z\right|^{2 m+1}\right) \\
& \quad+\varepsilon \sum_{j \text { good }} M^{2^{j}}\left(\sigma_{j} \operatorname{Re}\left(a_{j 0} z^{j}\right)-\frac{2 m}{\hat{C}}\left\|P^{j}\right\||z|^{j}\right)
\end{aligned}
$$

(The coefficients $a_{j 0}$ come from writing the $P^{\prime} s$ as in (2.16).) 
We omit the proof, since it is, except for some obvious small changes, the same as the proof of Lemma 4.5 in [7].

We introduce the notation $\mathcal{R}_{0}:=\{\rho(z)=r(0, z): r \in \mathcal{R}\}$. Using everything done in this section, we, finally, get

Lemma 4.11. Let $\delta>0$ be fixed as in Lemma 4.5 and choose the constant $L_{\delta}>0$ as in Lemma 4.4. Fix as the constant $\hat{C}$ from Lemma $4.7 \hat{C}:=\frac{8 m^{2}}{\delta}$ and let $M>1$ be chosen according to Lemma 4.7. For any small enough $\varepsilon>0$ define $\eta:=\frac{\varepsilon \delta}{8 m\left(1+C_{m}\right)}$ and then choose $A_{\eta}$ according to Lemma 4.8. Finally choose a constant $C>0$ such that

$$
\left|\rho-\sum_{j=2}^{2 m} P^{j}(z)\right|<C \quad \forall|z|<1
$$

for all $\rho \in \mathcal{R}_{0}$. Set

$$
R_{\varepsilon}:=\frac{\varepsilon M^{2^{j}} \delta\left\|P^{2 m}\right\|}{16 m\left(C+\left(1+C_{m}\right) A_{\eta}\right)}
$$

Then for all $\rho \in \mathcal{R}_{0}$

$$
\rho(z)+\varepsilon \sum_{j=2}^{2 m} M^{2^{j}} \sigma_{j} \operatorname{Re}\left(a_{j 0} z^{j}\right) \geq \varepsilon \sum_{j=2}^{2 m} \frac{M^{2^{j}} \delta}{16 m}\left\|P^{j}\right\||z|^{j} \quad \forall|z|<R_{\varepsilon}
$$

Proof. We just have to do the following chain of estimates

$$
\begin{aligned}
& \rho(z)+\varepsilon \sum_{j=2}^{2 m} M^{2^{j}} \sigma_{j} \operatorname{Re}\left(a_{j 0} z^{j}\right) \\
& =\sum_{j=2}^{2 m} P^{j}(z)+\eta \sum_{j=2}^{2 m}\left\|P^{j}\right\||z|^{j}+A_{\eta}|z|^{2 m+1}+\varepsilon \sum_{j=2}^{2 m} M^{2^{j}} \sigma_{j} \operatorname{Re}\left(a_{j 0} z^{j}\right) \\
& +\left(\rho-\sum_{j=2}^{2 m} P^{j}(z)\right)-\eta \sum_{j=2}^{2 m}\left\|P^{j}\right\||z|^{j}-A_{\eta}|z|^{2 m+1} \\
& \stackrel{\text { Lemma }}{{ }^{4.10}} C_{m}\left(\left.\left.\sum_{j \text { good }}\left|P^{j}(z)+\eta\left\|P^{j}\right\|\right| z\right|^{j}\left|+A_{\eta}\right| z\right|^{2 m+1}\right) \\
& +\varepsilon \sum_{j \operatorname{good}} M^{2^{j}}\left(\sigma_{j} \operatorname{Re}\left(a_{j 0} z^{j}\right)-\frac{2 m}{\hat{C}}\left\|P^{j}\right\||z|^{j}\right) \\
& -\left(C+A_{\eta}\right)|z|^{2 m+1}-\eta \sum_{j=2}^{2 m}\left\|P^{j}\right\||z|^{j} \\
& \geq C_{m}\left(\sum_{j \text { good }}\left|P^{j}(z)\right|+\frac{\varepsilon}{C_{m}} \sum_{j \text { good }} M^{2^{j}} \sigma_{j} \operatorname{Re}\left(a_{j 0} z^{j}\right)\right) \\
& -\left(C+A_{\eta}+C_{m} A_{\eta}\right)|z|^{2 m+1} \\
& -\left(\eta\left(1+C_{m}\right) \sum_{j=2}^{2 m}\left\|P^{j}\right\||z|^{j}+\frac{\varepsilon 2 m}{\hat{C}} \sum_{j=2}^{2 m} M^{2^{j}}\left\|P^{j}\right\||z|^{j}\right)
\end{aligned}
$$




$$
\begin{aligned}
& \underset{ }{\text { Lemma }}{ }^{4.6} C_{m}\left(\sum_{j \text { good }} \frac{\varepsilon M^{2^{j}}}{C_{m}} \delta\left\|P^{j}\right\||z|^{j}\right)-\left(C+A_{\eta}+C_{m} A_{\eta}\right)|z|^{2 m+1} \\
& -\left(\left.\eta\left(1+C_{m}\right) \sum_{j=2}^{2 m}\left\|P^{j}\right\|\right|^{j}+\frac{\varepsilon 2 m}{\hat{C}} \sum_{j=2}^{2 m} M^{2^{j}}\left\|P^{j}\right\||z|^{j}\right) \\
& \underset{\geq}{\operatorname{Lemma}} \sum_{j=2}^{2 m} \frac{\varepsilon M^{2^{j}} \delta}{2 m}\left\|P^{j}\right\||z|^{j}-\left(C+A_{\eta}+C_{m} A_{\eta}\right)|z|^{2 m+1} \\
& -\eta\left(1+C_{m}\right) \sum_{j=2}^{2 m}\left\|P^{j}\right\||z|^{j}-\frac{\varepsilon 2 m}{\hat{C}} \sum_{j=2}^{2 m} M^{2^{j}}\left\|P^{j}\right\||z|^{j} \\
\geq & \varepsilon \sum_{j=2}^{2 m} \frac{M^{2^{j}} \delta}{4 m}\left\|P^{j}\right\||z|^{j}-\left(C+\left(1+A_{\eta}\right)\right)|z|^{2 m+1}-\eta\left(1+C_{m}\right) \sum_{j=2}^{2 m}\left\|P^{j}\right\||z|^{j} \\
\geq & \varepsilon \sum_{j=2}^{2 m} \frac{M^{2^{j}} \delta}{8 m}\left\|P^{j}\right\||z|^{j}-\left(C+\left(1+C_{m}\right) A_{\eta}\right)|z|^{2 m+1}-\left(C+\left(1+C_{m}\right) A_{\eta}\right)|z|^{2 m+1} \\
\geq & \varepsilon \sum_{j=2}^{2 m} \frac{M^{2^{j}} \delta}{16 m}\left\|P^{j}\right\||z|^{j}+\left(\frac{\varepsilon M^{2^{j}} \delta}{16 m}\left\|P^{2 m}\right\||z|^{2 m}-\left(C+\left(1+C_{m}\right) A_{\eta}\right)|z|^{2 m+1}\right) \\
\geq & \varepsilon \sum_{j=2}^{2 m} \frac{M^{2^{j}}}{16 m}\left\|P^{j}\right\||z|^{j} \text { if }|z|<\frac{\varepsilon M^{2^{m}} \delta\left\|P^{2 m}\right\|}{16 m\left(C+\left(1+C_{m}\right) A_{\eta}\right)}
\end{aligned}
$$

This proves Lemma 4.11 as stated.

At this stage we want to come back to the original situation in $\mathbb{C}^{n}$ as described in section 2. Using the notations from there, we denote

$$
\hat{\mathcal{R}}:=\left\{r_{\zeta}: \zeta \in W_{0}\right\}
$$

Furthermore, we write the coordinates, to which the transformations $l_{\zeta}$ from Theorem 2.6 have already been applied, as $(w, z) \in \mathbb{C} \times \mathbb{C}^{n-1}$ instead of $\left(z_{1}, z^{\prime}\right)$ and put $u+i v=w$. Again, we use $\hat{m}=2 m$ is even and write out (2.13) for any $r_{\zeta} \in \mathcal{R}$ more explicitly as

$$
r_{\zeta}(w, z)=v+\sum_{j=2}^{2 m} P_{\zeta}^{j}(z)+u \sum_{l=1}^{m} Q_{\zeta}^{l}(z)+O\left(u^{2},|u||z|^{m+1},|z|^{2 m+1}\right)
$$

with $P_{\zeta}^{j}$ and $Q_{\zeta}^{l}$ homogeneous of order $j$. Notice that all coefficients of the $P_{\zeta}^{j}$ and the $Q_{\zeta}^{l}$ depend in a $\mathcal{C}^{\infty}$ way on $\zeta$. Furthermore, also the remainder terms are $\mathcal{C}^{\infty}$ in $\zeta$. Furthermore, for any $t \in T_{\zeta}^{10} \partial D \backslash\{0\}$, we have for $(w, z) \in \mathbb{C}^{2}$

$$
r_{\zeta, t}(w, z)=v+\sum_{j=2}^{2 m} P_{\zeta, t}^{j}(z)+u \sum_{j=1}^{m} Q_{\zeta, t}^{j}(z)+O\left(u^{2}, u z^{m+1}, z^{2 m+1}\right)
$$


where

$$
P_{\zeta, t}^{j}(z)=P_{\zeta}^{j}\left(w n_{\zeta}+z t\right), \quad Q_{\zeta, t}^{j}(z)=Q_{\zeta}^{j}\left(w n_{\zeta}+z t\right)
$$

As an immediate consequence of Lemma 4.11 we get in $\mathbb{C}^{n}$

Proposition 4.12. Let $W$ and $\eta_{0}$ be small enough. If $M>0$ is large enough and $\varepsilon>0$ is small enough, then there is a radius $R_{\epsilon}>0$ such that one has for all $r \in \mathcal{R}$

$$
r(0, z)+\epsilon \sum_{j=2}^{2 m} M^{2^{j}} \sigma_{j} \operatorname{Re}\left(\sum_{\substack{|\alpha|=j \\ \alpha=\left(\alpha_{1}, \ldots, \alpha_{n-1}\right)}} \frac{1}{\alpha !} \frac{\partial^{j} r(0)}{\partial z^{\alpha}} z^{\alpha}\right) \geq \epsilon \sum_{j=2}^{2 m} \frac{M^{2^{j}} \delta}{16 m}\left\|P_{t(z)}^{j}\right\||z|^{j}
$$

for all $z \in \mathbb{C}^{n-1},|z|<R_{\varepsilon}$. Here $P_{t(z)}^{j}(\tau)=P^{j}(\tau t)$ where $t \in \mathbb{C}^{n-1}$ is chosen in such a way that $z \in\{\tau t, \tau \in \mathbb{C}\}$ and $|t|=1$.

Proof. We work on $\hat{W}_{0}:=\left\{(\zeta, t) \in W_{0} \times \mathbb{C}^{n-1},|t|=1\right\}$ considering $t$ as an element of $T_{0}^{10} \partial D_{\zeta}$. We can apply Lemma 4.11 to a sufficiently small neighborhood $\mathcal{R}$ of any element $\rho_{0}(z)=r_{\zeta}(0, z \cdot t)$ with $(\zeta, t) \in \hat{W}_{0}$ and choose the constants of Lemma 4.11 accordingly. Notice, that a finite number of such neighborhoods $\mathcal{R}$ will suffice to deal with all points $(\zeta, t) \in \hat{W}_{0}$. We choose $M$ so large, and $\varepsilon$ and $R$ so small, that they work for all these neighborhoods. Then (4.13) follows directly from (4.10).

\section{Improving the Taylor series of the defining functions}

In order to carry over the estimates of Proposition 4.12 in $\mathbb{C}^{n-1}$ to estimates for the desired support functions in $\mathbb{C}^{n}$, we have to improve the coefficients in the Taylor series (4.11). More precisely, we have to eliminate the purely harmonic terms in the homogeneous polynomials $Q^{l}(z)$ for $l=1, \ldots, m$. This will be done by a finite sequence of simple coordinate transformations. Although they will destroy lineal convexity, the crucial estimate (4.13) will carry over to the new situation. We will show at first

Lemma 5.1. Let $W$ and $\eta_{0}$ be as in Proposition 4.12. There is for each $\zeta \in W_{0}=$ $\left\{\zeta \in W:|r(\zeta)| \leq \eta_{0}\right\}$ a biholomorphic coordinate transformation $(w, z)=$ $\Phi_{\zeta}(\tilde{w}, \tilde{z})$ on $W$ introducing the new coordinates $(\tilde{w}, \tilde{z})$ in the form $w=\tilde{w} \cdot(1-$ $\left.A_{\zeta}(z)\right), z=\tilde{z}$ with a holomorphic function $A_{\zeta}(z)$ depending in a $\mathcal{C}^{\infty}$ way on $\zeta$, $A_{\zeta}(0)=0$, such that the domains $\tilde{D}_{\zeta}:=\Phi^{-1}\left(D_{\zeta}\right)$ have defining functions $\tilde{r}=\tilde{r}_{\zeta}$ near $0 \in \tilde{W}:=\Phi^{-1}(W)$ of the form

$\tilde{r}(\tilde{w}, \tilde{z})=\tilde{v}+\sum_{j=2}^{2 m} P^{j}(\tilde{z})\left(1+O_{j}(\tilde{z})\right)+\tilde{u} \sum_{l=1}^{m} \hat{Q}^{l}(\tilde{z})+O\left(\tilde{u}^{2},|\tilde{u}||\tilde{z}|^{m+1},|\tilde{z}|^{2 m+1}\right)$ 
where the homogeneous terms $\hat{Q}^{l}$ of order $l$ do not contain any pluriharmonic terms and where the homogeneous parts $P^{j}$ are the same as for the original defining function $r_{\zeta}($ see (4.11)). In particular, the estimate (4.13) from Lemma 4.12 also holds for $\tilde{r}(0, \tilde{z})$.

Proof. We fix a $\zeta \in W_{0}$. The sum of all pluriharmonic terms of minimal degree, say $d$, in $\sum_{l=1}^{m} Q^{l}(z), Q^{l}$ as in (4.11), can be written as the real part of the holomorphic function

$$
A^{d}(z)=A_{\zeta}^{d}(z)=\sum_{|\alpha|=d} \frac{1}{\alpha !} \frac{\partial^{d+1} r_{\zeta}(0)}{\partial u \partial z^{\alpha}} z^{\alpha}=\sum_{|\alpha|=d} b_{\zeta, \alpha} z^{\alpha}
$$

With this we define a holomorphic coordinate transformation $(w, z)=\tilde{\Phi}^{d}(\tilde{w}, \tilde{z})$ by putting $z=\tilde{z}, w=\tilde{w} \cdot\left(1-i A^{d}(\tilde{z})\right)$. From this we get

$$
\begin{aligned}
& u=\tilde{u}+\tilde{u} \operatorname{Im} A^{d}(\tilde{z})+\tilde{v} \operatorname{Re} A^{d}(\tilde{z}) \\
& v=\tilde{v}-\tilde{u} \operatorname{Re} A^{d}(\tilde{z})+\tilde{v} \operatorname{Im} A^{d}(\tilde{z})
\end{aligned}
$$

After plugging this into (4.11) we get the following expression

$$
\begin{aligned}
r \circ \tilde{\Phi}(\tilde{w}, \tilde{z})= & \tilde{v}+\tilde{v} \operatorname{Im} A^{d}(\tilde{z})+\sum_{j=2}^{2 m} P^{j}(\tilde{z})+\tilde{u} \sum_{l=1}^{m} \hat{Q}^{l}(\tilde{z}) \\
& +\tilde{v}\left(\operatorname{Re} A^{d}(\tilde{z})\right) \sum_{l=1}^{m} Q^{l}(\tilde{z}) \\
& +O\left(\tilde{u}^{2}, \tilde{u} \tilde{v} \operatorname{Re} A^{d}(\tilde{z}), \tilde{v}^{2},|\tilde{u}||\tilde{z}|^{m+1},|\tilde{v}||z|^{m+1+d},|z|^{2 m+1}\right)
\end{aligned}
$$

where the $\hat{Q}^{l}$ are new homogeneous functions of degree $l$ with the property, that their lowest order pluriharmonic terms have degree $>d$.

Notice next, that the boundary $\partial D_{\zeta}$ can again be written in the new coordinates $(\tilde{w}, \tilde{z})$ as a graph over $\{\tilde{v}=0\}$ near 0 , namely, as the zero set of a new function $\tilde{r}(\tilde{w}, \tilde{z})$ of the form

$$
\tilde{r}=\tilde{v}+\sum_{j=2}^{2 m} \lambda_{j}(\tilde{z})+\tilde{u} \sum_{l=1}^{m} \mu_{l}(\tilde{z})+O\left(\tilde{u}^{2},|\tilde{u}||\tilde{z}|^{m+1},|\tilde{z}|^{2 m+1}\right)
$$

On $\tilde{r}=0$ we also have $r \circ \Phi(\tilde{w}, \tilde{z})=0$. Hence we can solve (5.2) for $\tilde{v}$ and can plug the result into (5.1) obtaining the identity

$$
\begin{aligned}
0=[ & \left.-\sum_{j=2}^{2 m} \lambda_{j}(\tilde{z})-\tilde{u} \sum_{l=1}^{m} \mu_{l}(\tilde{z})\right]\left[1+\operatorname{Im} A^{d}(\tilde{z})+\operatorname{Re} A^{d}(\tilde{z}) \sum_{l=1}^{m} Q^{l}(\tilde{z})\right] \\
& +\sum_{j=2}^{2 m} P^{j}(\tilde{z})+\tilde{u} \sum_{l=1}^{m} \hat{Q}^{l}(\tilde{z})+\sum_{j=2}^{2 m} \lambda_{j}(\tilde{z}) O_{j}(|\tilde{z}|) \\
& +\tilde{u} \sum_{l=1}^{m} \mu_{l}(\tilde{z}) O_{l}(|\tilde{z}|)+O\left(\tilde{u}^{2},|\tilde{u}||\tilde{z}|^{m+1},|\tilde{z}|^{2 m+1}\right)
\end{aligned}
$$

By comparing terms we get from this the equations

$$
\begin{gathered}
O\left(|\tilde{z}|^{2 m+1}\right)-\sum_{j=2}^{2 m} \lambda_{j}(\tilde{z})\left(1+O_{j}(|\tilde{z}|)\right)+\sum_{j=2}^{2 m} P^{j}(\tilde{z})=0 \\
\tilde{u} \cdot O\left(|\tilde{z}|^{m+1}\right)-\tilde{u} \sum_{l=1}^{m} \mu_{l}(\tilde{z})\left(1+O_{l}(|\tilde{z}|)\right)+\sum_{l=1}^{m} \hat{Q}(\tilde{z})=0
\end{gathered}
$$


Hence the defining function $\tilde{r}$ has the form

$$
\begin{aligned}
\tilde{r}=\tilde{v} & +\sum_{j=2}^{2 m} P^{j}(z)\left(1+O_{j}(|\tilde{z}|)\right)+\tilde{u} \sum_{l=1}^{m} \hat{Q}^{l}(\tilde{z})\left(1+O_{l}(|\tilde{z}|)\right) \\
& +O\left(\tilde{u}^{2},|\tilde{u}||\tilde{z}|^{m-1},|\tilde{z}|^{2 m+1}\right)
\end{aligned}
$$

Notice now, that $\sum_{l=1}^{m} \hat{Q}_{l}(\tilde{z})\left(1+O_{l}(|\tilde{z}|)\right)$ does no longer contain any pluriharmonic terms of degree $\leq d$. After redefining the $P^{j}$ and the $Q^{l}$ such that in the new coordinates, which we denote again by $w=u+i v, z$, we have as before

$$
\tilde{r}=v+\sum_{j=2}^{2 m} P^{j}(z)+u \sum_{l=1}^{m} Q^{l}(z)+O\left(u^{2},|u||z|^{m+1},|z|^{2 m+1}\right)
$$

but now with $\sum_{l=1}^{m} Q^{l}(z)$ without pluriharmonic terms of degree $\leq d$, we can repeat the same procedure inductively until and including $d=m$. Keeping track of the changes made at each step proves the Lemma.

It is very important for us to observe, that, although the coordinate transformations of Lemma 5.1 will, in general, destroy lineal convexity, the estimate (4.13) from Proposition 4.12 remains true in the new coordinates with the new defining functions. We have

Corollary 5.2. Let the coordinates $(\tilde{w}, \tilde{z})$ and the defining function $\tilde{r}(\tilde{w}, \tilde{z})$ corresponding to $r(w, z)$ be chosen as in Lemma 5.1. Then one has in analogy to (4.13) the estimate

$$
\tilde{r}(0, \tilde{z})+\varepsilon \sum_{j=2}^{2 m} M^{2^{j}} \sigma_{j} \operatorname{Re}\left(\sum_{\substack{|\alpha|=j \\ \alpha=\left(\alpha_{1}, \ldots, \alpha_{n-1}\right)}} \frac{1}{\alpha !} \frac{\partial^{j} r(0)}{\partial \tilde{z}^{\alpha}}\right) \geq \varepsilon \sum_{j=2}^{2 m} \frac{M^{2^{j}} \delta}{16 m}\left\|P_{t(\tilde{z})}^{j}\right\||\tilde{z}|^{j}
$$

for all $\tilde{z} \in \mathbb{C}^{n-1},|\tilde{z}|<R_{\varepsilon}$. Here $t(\tilde{z})$ has been chosen in such a way that $\tilde{z} \in$ $\{\tau t(\tilde{z}): \tau \in \mathbb{C}\}$ and $|t(\tilde{z})|=1$. Furthermore,

$$
P^{j}(z)=\sum_{|\alpha|+|\beta|=j} \frac{1}{\alpha ! \beta !} \frac{\partial^{j} r(0)}{\partial z^{\alpha} \partial \bar{z}^{\beta}} z^{\alpha} \bar{z}^{\beta}
$$

and $P_{t(\tilde{z})}^{j}(\tau)=P^{j}(\tau t(\tilde{z}))$.

Proof. We write in total analogy to (4.11) the homogeneous decomposition

$$
\tilde{r}(\tilde{w}, \tilde{z})=\tilde{v}+\sum_{j=2}^{2 m} \hat{P}^{j}(\tilde{z})+\tilde{u} \sum_{l=1}^{m} \hat{Q}(\tilde{z})+O\left(\tilde{u}^{2},|\tilde{u}||\tilde{z}|^{m+1},|\tilde{z}|^{2 m+1}\right)
$$


Then we get from Lemma 5.1

$$
\hat{P}^{j}(\tilde{z})=P^{j}(\tilde{z})+\sum_{2 \leq i<j} P^{i}(\tilde{z}) h_{i}^{j}(\tilde{z})
$$

with functions $h_{i}^{j}$ homogeneous of order $j-i$. Furthermore, the $\hat{Q}(\tilde{z})$ do not contain pluriharmonic terms. Next, we observe from the proof of Proposition 4.12, that it suffices to show that estimate (4.10) from Lemma 4.11 holds for the restrictions $\tilde{\rho}(z):=\tilde{r}(0, z t), z \in \mathbb{C}$, for any $t \in \mathbb{C}^{n-1},|t|=1$. As follows from (5.5) they have the form

$$
\tilde{\rho}(z)=\sum_{j=2}^{2 m} \hat{P}^{j}(z)+O\left(z^{2 m+1}\right)
$$

where, for simplicity, we have written $\hat{P}^{j}(z)$ instead of $\hat{P}(z t)$. From (5.6) we also get for the purely holomorphic part of $\hat{P}^{j}(z)$ as compared to the holomorphic part of $P^{j}(z)$ the estimate

$$
\operatorname{Re} \hat{a}_{j 0} z^{j}=\operatorname{Re} a_{j 0} z^{j}+O\left(\sum_{2 \leq i<j}\left\|P^{i}\right\||z|^{j}\right)
$$

We will, however, not need this in the following.

By putting (5.6) into (4.10) we can estimate

$$
\begin{aligned}
& \sum_{j=2}^{2 m} \hat{P}^{j}(z)+\varepsilon \sum_{j=2}^{2 m} M^{2^{j}} \sigma_{j} \operatorname{Re}\left(a_{j 0} z^{j}\right) \\
& \quad \geq \sum_{j=2}^{2 m} P^{j}(z)+\varepsilon \sum_{j=2}^{2 m} M^{2^{j}} \sigma_{j} \operatorname{Re}\left(a_{j 0} z^{j}\right)-C \sum_{j=2}^{2 m} \sum_{2 \leq i<j}\left\|P^{i}\right\||z|^{j} \\
& \quad \stackrel{(4.10)}{\geq} \varepsilon \sum_{j=2}^{2 m} \frac{M^{2^{j}} \delta}{16 m}\left\|P^{j}\right\||z|^{j}-C \sum_{j=2}^{2 m} \sum_{2 \leq i<j}\left\|P^{i}\right\||z|^{j} \\
& \quad \geq \varepsilon \sum_{j=2}^{2 m} \frac{M^{2^{j}} \delta}{16 m}\left\|P^{j}\right\||z|^{j}
\end{aligned}
$$

for all $z \in \mathbb{C}$ with $|z| \leq R_{\varepsilon}$ (after possibly shrinking $R_{\varepsilon}$ uniformly in $\tilde{\rho}$ ). Notice, that the last inequality in this chain holds, since in the second term of the previous line all $\left\|P^{i}\right\|$ appear for $i<2 m$, however always with the power $|z|^{j}$ as factor which is higher than the power $|z|^{i}$ appearing with the same factor $\left\|P^{i}\right\|$ in the first term.

\section{Inequalities in $\mathbb{C}^{2}$}

Again as in [7] we next want to get the decisive estimates on the desired support functions in $\mathbb{C}^{2}$. Since, at this point, in [7] we strongly used the convexity of the given domains, we have to replace those arguments. In fact, as it turns out, it is just the pseudoconvexity of lineally convex domains which suffices for our purposes. 
In this situation in $\mathbb{C}^{2}$ we choose coordinates, now again called $(w, z) \in \mathbb{C}^{2}$, and a defining function $\tilde{r}(w, z)$ as in Corollary 5.2. We decompose it into homogeneous terms in the form

$$
\tilde{r}(w, z)=v+\sum_{j=2}^{2 m} \hat{P}^{j}(z)+u \sum_{l=1}^{m} \hat{Q}^{l}(z)+O\left(u^{2}, u z^{m+1}, z^{2 m+1}\right)
$$

and assume, that the $\hat{Q}^{l}$ do not contain any harmonic terms (we may do so according to Lemma 5.1). In particular, we need to sum in the second term of $\tilde{r}$ only from $l=2$. The pseudoconvexity of our original domains, which, of course, is not destroyed by the coordinate transformations used in Lemma 5.1, tells us, that the function $A$ from (3.2) being the Levi form of the level sets of $\tilde{r}$ is $\geq 0$. Therefore, formula (3.12) gives us

$$
\begin{aligned}
0 \leq & A(u, z) \\
\leq & C\left(u^{2}+|u||z|^{m-1}+|z|^{2 m-1}+\sum_{j=2}^{2 m}\left\|P^{j}\right\||z|^{l-1}+|u| \sum_{l=2}^{m}\left\|Q^{j}\right\||z|^{j-1}\right) \\
& +\frac{1}{4} \sum_{l=2}^{2 m} P_{z \bar{z}}^{l}+\frac{u}{4} \sum_{l=2}^{m} Q_{z \bar{z}}^{l}
\end{aligned}
$$

on a certain neighborhood of 0 , independent of the choice of $\tilde{r}$. From this we immediately get

$$
\left|\frac{u}{4} \sum_{l=2}^{m} Q_{z \bar{z}}^{l}\right| \leq C\left(\sum_{j=2}^{2 m}\left\|P^{j}\right\||z|^{j-2}+|u|^{2}+|u| \sum_{l=2}^{m}\left\|Q^{j}\right\||z|^{j-1}\right)
$$

since $\left|u z^{m-1}\right| \lesssim 2|u|^{2}+\left\|P^{2 m}\right\||z|^{2 m-2}$. By putting for any given $z \neq 0$ the variable $u:=\left(\sum_{j=2}^{2 m}\left\|P^{j}\right\||z|^{j-2}\right)^{1 / 2}$ we, finally, get

Lemma 6.1. For the decomposition (6.1) one has for a small enough number $d>0$

$$
\left(\sum_{j=2}^{m} Q_{z \bar{z}}^{j}\right)^{2} \leq C \sum_{j=2}^{2 m}\left\|P^{j}\right\||z|^{j-2}+C\left(\sum_{j=2}^{m}\left\|Q^{j}\right\||z|^{j-1}\right)^{2}
$$

for all $z$ with $|z| \leq d$.

(Notice, that lineal convexity has not been used in proving (6.3).)

As a corollary of this Lemma we will derive from (6.3) an estimate of the $Q$ 's against the $P$ 's. Namely we have

Lemma 6.2. In the situation established at the beginning of this section there is a constant $C>0$, such that, after possibly shrinking $d>0$,

$$
\sum_{i=2}^{m}\left\|Q^{i}\right\||z|^{i} \leq C \sqrt{\sum_{l=2}^{2 m}\left\|P^{l}\right\||z|^{l}|z|}
$$

and, hence,

$$
\left|u \sum_{i=2}^{m} Q^{i}\right| \leq u^{2}+C^{2}|z|^{2} \sum_{l=2}^{2 m}\left\|P^{l}\right\||z|^{l}
$$

for all $|z|<d$. 
Proof. The proof consists of two parts.

1) Let $L>0$ be large enough (how large it has to be, will be specified later) and suppose, there is a number $a>0$ and an index $j, 2 \leq j \leq m$, such that one has for all $z \in \mathbb{C}$ with $a<|z|<L a$ the estimates

$$
\left\|Q^{j}(z)\right\||z|^{j} \geq\left\|Q^{i}(z)\right\||z|^{i} \quad \forall i \neq j
$$

Write $Q^{j}(z)=\sum_{l=1}^{j-1} b_{l}^{j} z^{l} \bar{z}^{j-l}$ and pick an index $l$ such that $\left|a_{l}^{j}\right| \geq \frac{1}{j}\left\|Q^{j}\right\|$. Consider any $z$ with $|z|=a \sqrt{L}$. From the fact, that (6.5) even holds for $|z|=a$, one obtains for $|z|=a \sqrt{L}$ and $j \geq i$

$$
\frac{\left\|Q^{j}\right\||z|^{j-2}}{L^{\frac{j-i}{2}}} \geq\left\|Q^{i}\right\||z|^{i-2}
$$

and, hence,

$$
\frac{1}{\sqrt{L}}\left\|Q^{j}\right\||z|^{j-2} \geq\left\|Q^{i}\right\||z|^{i-2}
$$

for $j>i$. By starting with (6.5) on $\{|z|=L a\}$ the inequality also follows for $i>j$, if $|z|=a \sqrt{L}$. With this one obtains

$$
\begin{aligned}
& \left|a_{l}^{j}\right||z|^{j-2} \\
& \quad=\frac{1}{2 \pi}\left|\int_{0}^{2 \pi} a_{l}^{j} z^{l-1} \bar{z}^{j-l-1} e^{i(j-2 l) \theta} d \theta\right| \\
& \quad=\left|\frac{1}{2 \pi} \int_{0}^{2 \pi} \frac{1}{l(j-l)} \frac{\partial^{2}}{\partial z \partial \bar{z}} a_{l}^{j} z^{l} \bar{z}^{j-l} e^{i(j-2 l) \theta} d \theta\right| \\
& \quad=\frac{1}{2 \pi}\left|\int_{0}^{2 \pi} \frac{1}{l(j-l)} Q_{z \bar{z}}^{j} e^{i(j-2 l) \theta} d \theta\right| \\
& \quad \leq \frac{1}{2 \pi l(j-l)}\left|\int_{0}^{2 \pi} \sum_{k=2}^{m} Q_{z \bar{z}}^{k} e^{i(j-2 l) \theta} d \theta\right|+C \sum_{i \neq j}\left\|Q^{i}\right\||z|^{i-2} \\
& \quad(6.3) \text { and (6.6) } \\
& \quad \leq \sqrt{\sum_{k=2}^{2 m}\left\|P^{k}\right\||z|^{k-2}}+\frac{m}{\sqrt{L}} C\left\|Q^{j}\right\||z|^{j-2}+C \sum_{i}\left\|Q^{i}\right\||z|^{i-1}
\end{aligned}
$$

We assume now, more precisely, that $L$ has been chosen such that $\frac{m}{\sqrt{L}} C<\frac{1}{2 m}$. Then it follows together with the choice of $l$ that

$$
\left\|Q^{j}\right\||z|^{j-2} \leq C \sqrt{\sum_{k=2}^{2 m}\left\|P^{k}\right\||z|^{k-2}}
$$

and hence

$$
\left\|Q^{i}\right\||z|^{i-2} \leq C \sqrt{\sum_{k=2}^{2 m}\left\|P^{k}\right\||z|^{k-2}}
$$

for all $i$ and $|z|=a \sqrt{L}$. Notice, however, that this implies, that, after changing the constant $C$, one even has the same inequality (6.8) for all $z$ with $\frac{a}{L^{m}}<|z|<$ $a L^{m}$. 
2) Let now, for any $\tilde{r}$ as considered in this section, $j$ be the minimal index between 2 and $m$ such that $Q^{j} \neq 0$. Then it follows, that (6.5) holds with this $j$ on a small disc $\Delta_{\eta}=\Delta(0, \eta)$ around 0 . Hence, (6.8) has been proved on $\Delta_{\eta}$ (notice, however, that $\eta$ cannot be chosen uniformly in $\tilde{r}$ ). Let now $d$ be as in Lemma 6.2. Then there is for any radius $R, \eta \leq R \leq d$, a $j$ such that (6.5) holds for $|z|=R$ and, as can easily be seen, these indices $j$ are monotonously increasing in $R$. Hence, there are radii $\eta=R^{1}<\cdots<R^{k}=d$ with $k<m$, such that on each annulus $A_{i}:=A_{R^{i-1}, R^{i}}:=\left\{R^{i-1} \leq|z| \leq R^{i}\right\}$ one $Q^{j}$ is dominating in the sense of (6.5). We call such an annulus $A_{i}$ thick, if $\frac{R^{i}}{R^{i-1}} \geq L$. We consider 1st case: There is no thick annulus. - Then, since $\bigcup A_{i}=A_{\eta, d}$ and since there are at most $m$ annuli, we have $d \leq L^{m} \eta$, hence (6.8) holds on $\Delta(0, d)$.

2nd case: there are thick annuli. - Let $A_{i}$ be any of them and suppose there is even a next one, say $A_{j}$ for some $j>i$. Then, since $j-i<m$, we have $R^{j-1} \leq L^{m} R^{i}$. Hence (6.8) does not only hold on $A_{i}$ but even all the way up to the radius $R^{j-1}$. In case, there is no next thick annulus after $A_{i}$, we get (6.8) up to the radius $d$.

This proves the Lemma.

We now can prove an estimate which is essentially what we want to show in Theorem 2.6 for the 2-dimensional slices $D_{\zeta, t}$. Namely, we have

Proposition 6.3. Let $W$ and $\eta_{0}$ as introduced in section 2 be small enough. Let $\delta>0$ be small enough (chosen according to Lemma 4.5). Choose numbers $M>0$ large enough and $\varepsilon>0$ small enough. Denote by $\tilde{\mathcal{R}}$ the set of all defining functions $\tilde{r}$ obtained from the set $\mathcal{R}$ after the coordinate changes of Lemma 5.1 have been made. Choose another number $K>0$ large enough. Then there is a radius $R_{\mathcal{E}}$ such that the following holds true: if one puts for any $\tilde{r} \in \tilde{\mathcal{R}}$

$$
S_{\tilde{r}}(w, z):=\frac{w}{i}-K w^{2}-\varepsilon \sum_{j=2}^{2 m} M^{2^{j}} \frac{1}{j !} \sigma_{j} \frac{\partial^{j} \tilde{r}(0)}{\partial z^{j}} z^{j}
$$

one has for $(w, z) \in B\left(0, R_{\varepsilon}\right)$ the estimate

$$
\operatorname{Re}\left(S_{\tilde{r}}(w, z)\right) \leq \tilde{r}(w, z)(1+K \tilde{r}(w, z))-\frac{K}{2} u^{2}-\frac{\varepsilon}{2} \sum_{j=2}^{2 m} \frac{M^{2^{j}} \delta}{16 m}\left\|P^{j}\right\||z|^{j}
$$

where, of course,

$$
P^{j}(z)=\sum_{k+l=j} \frac{1}{k ! l !} \frac{\partial^{j} \tilde{r}(0)}{\partial z^{k} \partial \bar{z}^{l}} z^{k} \bar{z}^{l}
$$

for $j=2, \ldots, 2 m$.

Remark 6.4. If $\tilde{r} \in \tilde{\mathcal{R}}$ is the defining function associated with $r \in \mathcal{R}$ via the respective coordinate transformation of Lemma 5.1, then one also has

$$
P^{j}(z)=\sum_{k+l=j} \frac{1}{k ! l !} \frac{\partial^{j} r(0)}{\partial z^{k} \partial \bar{z}^{l}} z^{k} \bar{z}^{l}
$$

as already mentioned in Lemma 5.1. 
Proof. It follows from (6.9), that one has

$$
\operatorname{Re} S_{\tilde{r}}(w, z)=v-K u^{2}+K v^{2}-\varepsilon \sum_{j=2}^{2 m} M^{2^{j}} \sigma_{j} \operatorname{Re}\left(a_{j 0} z^{j}\right)
$$

if, as usual, $a_{j 0}:=\frac{1}{j !} \frac{\partial^{j} \tilde{r}(0)}{\partial z^{j}}=\frac{1}{j !} \frac{\partial^{j} r(0)}{\partial z^{j}}$. In order to estimate this we substitute at first for $v$ what we get from (6.1), namely,

$$
v=\tilde{r}(w, z)-\tilde{r}(0, z)-u \sum_{j=1}^{m} Q^{j}(z)+O\left(u^{2}, u z^{m+1}, z^{2 m+1}\right)
$$

Then we get from (6.11) the estimate

$$
\begin{aligned}
\operatorname{Re} & S_{\zeta}(w, z) \\
= & O\left(u^{2}, u z^{m+1}, z^{2 m+1}\right)+\left(\tilde{r}(w, z)-\tilde{r}(0, z)-u \sum_{l=2}^{m} Q^{l}(z)\right)-K u^{2} \\
& +K\left(\tilde{r}(w, z)-\sum_{j=2}^{2 m} P^{j}(z)-u \sum_{l=2}^{m} Q^{l}(z)\right)^{2}-\varepsilon \sum_{j=2}^{2 m} M^{2^{j}} \sigma_{j} \operatorname{Re}\left(a_{j 0} z^{j}\right) \\
\leq & O\left(u^{2}, u z^{m+1}, z^{2 m+1}\right)+\tilde{r}(w, z)(1+K \tilde{r}(z, w)) \\
& -\left[\tilde{r}(0, z)+\varepsilon \sum_{j=2}^{2 m} M^{2^{j}} \sigma_{j} \operatorname{Re}\left(a_{j 0} z^{j}\right)\right]-u \sum_{l=2}^{m} Q^{l}(z)-K u^{2} \\
& +K C\left(\sum_{j=2}^{2 m}\left\|P^{j}\right\||z|^{j+2}+|u| \sum_{l=2}^{m}\left\|Q^{l}\right\||z|^{l+2}+|\tilde{r}(w, z)| \sum_{j=2}^{2 m}\left\|P^{j}\right\||z|^{j}\right) \\
& +K C\left|\tilde { r } ( w , z ) \left\|\left.u\left|\sum_{l=2}^{m}\left\|Q^{l}\right\|\right| z\right|^{l}\right.\right.
\end{aligned}
$$

By using Corollary 5.2 and Lemma 6.2 we can after choosing $R_{\varepsilon}$ small enough, uniformly in $\tilde{r}$, proceed to

$$
\begin{aligned}
\operatorname{Re} & S_{\tilde{r}}(w, z) \\
\leq & O\left(u^{2}, u z^{m+1}, z^{2 m+1}\right)+\tilde{r}(w, z)(1+K \tilde{r}(w, z))-\varepsilon \sum_{j=2}^{2 m} \frac{M^{2^{j}} \delta}{16 m}\left\|P^{j}\right\||z|^{j} \\
& +\left(1+K C|z|^{2}+|\tilde{r}(w, z)|\right)\left[u^{2}+C^{2}|z|^{2} \sum_{l=2}^{2 m}\left\|P^{l}\right\||z|^{l}\right]-K u^{2} \\
& +K C\left(|z|^{2}+|\tilde{r}(w, z)|\right) \sum_{j=2}^{2 m}\left\|P^{j}\right\||z|^{j} \\
& +\tilde{r}(w, z)(1+K \tilde{r}(w, z))-\frac{1}{2} K u^{2}-\frac{\varepsilon}{2} \sum_{j=2}^{2 m} \frac{M^{2 j} \delta}{16 m}\left\|P^{j}\right\||z|^{j}
\end{aligned}
$$

This proves the Proposition.

\section{Proof of Theorems 2.2 and Theorem 2.6}

Notice, that Proposition 6.3 says essentially the same as Theorem 2.6 for $n=2$, the only difference being, that in Proposition 6.3 the defining functions are used, which represent $\partial D$ in the coordinates given by Lemma 5.1 as a graph over the real tangent plane (these defining functions were called $r_{\zeta}^{g}$ in (2.13)), whereas in Theorem 2.6 we allow arbitrary defining functions in these coordinates. So, in order to complete the proof of Theorem 2.6 we still have two tasks:

1. We need to generalize Proposition 6.3 to arbitrary dimensions;

2. we need to replace the special defining functions by arbitrary ones. 
Generalizing Proposition 6.3 to arbitrary dimension is straightforward. Namely, we make all hypotheses of Theorem 2.6 and choose the coordinates as described in that Theorem. We take as defining function $\tilde{r}_{\zeta}(\tilde{z}):=\tilde{r}_{\zeta}\left(\tilde{z}_{1}, \tilde{z}^{\prime}\right)$ the corresponding function $\tilde{r}_{\zeta}^{g}(\tilde{z})$ from (2.13) and we define for every $\zeta \in W_{0}$ the function $S_{\zeta}(\tilde{z})$ by the formula (2.10). Now, we just have to notice, what has been observed already in Remark 2.3, namely, for any unit vector $t=\left(0, t_{2}, \ldots, t_{n}\right)$ we get after writing $\tilde{r}_{\zeta, t}(\tilde{w}):=\tilde{r}_{\zeta}\left(\tilde{w}_{1}(i, 0, \ldots, 0)+\tilde{w}_{2} t\right)$

$$
\frac{1}{j !} \frac{\partial^{j} \tilde{r}_{\zeta, t}(0)}{\partial \tilde{w}_{2}^{j}} \tilde{w}_{2}^{j}=\sum_{|\alpha|=j} \frac{1}{\alpha !} \frac{\partial^{j} \tilde{r}_{\zeta}(0)}{\partial \tilde{z}^{\prime \alpha}} t^{\prime \alpha} \tilde{w}_{2}^{j}
$$

and

$$
\tilde{P}_{\zeta, t}^{j}\left(\tilde{w}_{2}\right)=\sum_{|\alpha|+|\beta|=j} \frac{1}{\alpha !} \frac{1}{\beta !} \frac{\partial^{j} \tilde{r}_{\zeta}(0)}{\partial \tilde{z}^{\prime \alpha} \partial \overline{\tilde{z}}^{\prime}} t^{\prime \alpha}{\overline{t^{\prime}}}^{\beta} \tilde{w}_{2}^{|\alpha|} \tilde{\tilde{w}}_{2}^{|\beta|}
$$

With this the estimate (2.12) follows directly from (6.10), such that task 1 from above has been done.

In order to get task 2 done, we again make all hypotheses as in Theorem 2.6 and let $\tilde{r}_{\zeta}(\tilde{z})$ be defined as there starting with an arbitrary defining function $r$ of $D$. Let us denote for a moment by $r_{\zeta}^{g}(\tilde{z})$ the defining function representing $D$ in the $\tilde{z}$-coordinates as a graph as just used in treating task 1, in particular, in (7.1) and (7.2). Then there is a strictly positive function $\tilde{h}_{\zeta}(\tilde{z}), \mathcal{C}^{\infty}$ in $(\tilde{z}, \zeta)$, such that we have

$$
\tilde{r}_{\zeta}^{g}(\tilde{z})=\tilde{r}_{\zeta}(\tilde{z}) \tilde{h}_{\zeta}(\tilde{z})
$$

We denote for a moment by $\tilde{P}_{\zeta, t}^{g, j}\left(\tilde{w}_{2}\right)$ the homogeneous polynomial from (7.2) obtained by using $\tilde{r}_{\zeta}^{g}(\tilde{z})$ and by $\tilde{P}_{\zeta, t}^{j}\left(\tilde{w}_{2}\right)$ the one obtained from $\tilde{r}_{\zeta, t}\left(\tilde{w}_{2}\right)$. Then (7.3) immediately gives the following estimates:

$$
\sum_{|\alpha|=j} \frac{1}{\alpha !} \frac{\partial^{j} \tilde{r}_{\zeta}^{g}(0)}{\partial \tilde{z}^{\prime \alpha}} t^{\prime \alpha} \tilde{w}_{2}^{j}=\tilde{h}_{\zeta}(0) \sum_{|\alpha|=j} \frac{1}{\alpha !} \frac{\partial^{j} \tilde{r}_{\zeta}(0)}{\partial \tilde{z}^{\prime \alpha}} t^{\prime \alpha} \tilde{w}_{2}^{j}+O\left(\sum_{l<j}\left\|\tilde{P}_{\zeta, t}^{g, l}\right\|\left|\tilde{w}_{2}\right|^{l+1}\right)
$$

and

$$
\begin{aligned}
& \sum_{|\alpha|+|\beta|=j} \frac{1}{\alpha !} \frac{1}{\beta !} \frac{\partial^{j} \tilde{r}_{\zeta}^{g}(0)}{\partial \tilde{z}^{\prime \alpha} \partial \overline{\tilde{z}^{\prime}} \beta} t^{\prime \alpha}{\overline{t^{\prime}}}^{\beta} \tilde{w}_{2}^{|\alpha|} \overline{\tilde{w}}_{2}^{|\beta|} \\
& =\tilde{h}_{\zeta}(0) \sum_{|\alpha|+|\beta|=j} \frac{1}{\alpha !} \frac{1}{\beta !} \frac{\partial^{j} \tilde{r}_{\zeta}(0)}{\partial \tilde{z}^{\prime \alpha} \partial{\overline{\tilde{z}^{\prime}}}^{\beta}} t^{\prime \alpha}{\overline{t^{\prime}}}^{\beta} \tilde{w}_{2}^{|\alpha|} \tilde{\tilde{w}}_{2}^{|\beta|} \\
& \quad+O\left(\sum_{l<j}\left\|\tilde{P}_{\zeta, t}^{g, l}\right\|\left|\tilde{w}_{2}\right|^{l+1}\right)
\end{aligned}
$$


From this we get

$$
\begin{aligned}
\sum_{l=2}^{2 m}\left\|\tilde{P}_{\zeta, t}^{l}\right\|\left|\tilde{w}_{2}\right|^{l} & \leq \sum_{f_{l=2}}^{2 m}\left(\left\|\tilde{P}_{\zeta, t}^{g, l}\right\|\left|\tilde{w}_{2}\right|^{l}+C_{l}\left\|\tilde{P}_{\zeta, t}^{g, l}\right\|\left|\tilde{w}_{2}\right|^{l+1}\right) \\
& \lesssim \sum_{l=2}^{2 m}\left\|\tilde{P}_{\zeta, t}^{g, l}\right\|\left|\tilde{w}_{2}\right|^{l}
\end{aligned}
$$

if $\left|\tilde{w}_{2}\right|$ is small enough. The analogous estimate, where the role of $\tilde{P}_{\zeta, t}^{l}$ and $\tilde{P}_{\zeta, t}^{g, l}$ is just interchanged, also holds.

If we plug all this into (2.10) and (2.12) replacing thereby the defining functions $\tilde{r}_{\zeta}^{g}$ by $\tilde{r}_{\zeta}$ we get Theorem 2.6 in full generality (the calculations are similar to what has been done in proving Corollary 5.2).

It remains to show how Theorem 2.2 follows from Theorem 2.6, more precisely, how to derive estimate (2.6) from estimate (2.12). For this we drop at first the term $-\frac{K}{2}\left(\operatorname{Re} \tilde{w}_{1}\right)^{2}$ from (2.12). This does not destroy the estimate. After this we just have to return to the original $z$-coordinates. We do this in two steps. Namely, at first, we return to the coordinates denoted by $\hat{z}$ in Theorem 2.6. This, however, does not cause any difficulties, since, first of all, $\tilde{\Phi}_{\zeta}\left(0, \tilde{z}_{2}, \ldots, \tilde{z}_{n}\right)=\left(0, \tilde{z}_{2}, \ldots, \tilde{z}_{n}\right)$ and since, secondly, as Corollary 5.2 shows, returning to the $\hat{z}$-coordinates just means returning from the inequality (5.2) to the original inequality (4.13) from which (5.2) had been derived. The second step, namely returning from the $\hat{z}$-ccordinates to the original $z$-ccordinates is trivial, since this coordinate change is affine unitary. This finishes the proof of Theorem 2.2.

\section{Possible applications}

The $\mathcal{C}^{\infty}$-family of support functions constructed in [7] for convex domains of finite type meanwhile has found many applications. However, for all of them another tool was quite important, namely, the machinery of non-isotropic pseudoballs and a corresponding metric on such domains. In his thesis (in preparation) Michael Conrad, University of Wuppertal, now has developed this machinery also for lineally convex domains of finite type. Therefore, it can be expected, that Theorems 2.2 and 2.6 are the basis for generalizing the following results from convex domains of finite type to lineally convex domains of finite type:

1. Hölder and $L^{p}$-estimates for solution operators of Andersson-Berndtsson type for the $\bar{\partial}$-equations (see the totally analogous work in the convex case [6], [12], [14]);

2. Characterization of the zero sets of the Nevanlinna class (see [11]).

Many other obvious questions of quantitative complex analysis on our domains can be treated. Let us mention as an example the question of the precise subellipticity gain for the $\bar{\partial}$-Neumann-problem on lineally convex domains of finite type.

Given the fact, that there are many critical counter-examples (see [21], [23], [22], [8], [9]), but also a recent positive result for convex domains (see [10], based 
on [7]), it also might be very interesting to find out, what the situation is regarding the possibility of $L^{\infty}$-bounded holomorphic extension of bounded holomorphic functions on certain complex submanifolds of lineally convex domains of finite type. We do not pursue this matter here.

As already said, it can be expected, that most of these applications will be more or less routine based on the corresponding work for the convex finite type case. Only where convexity has been used in an additional way except for the pseudometric and the existence of the support functions with good estimates (like, for instance, in [10]), new ideas will be required.

Acknowledgements. This article was written while the first named author was visiting the Department of Mathematics of the University of Michigan, Ann Arbor, as a F. Gehring Visiting Professor within the framework of the Fred and Lois Gehring Special Year in Complex Analysis. He would like to express his sincere thanks for this opportunity for doing research in a stimulating atmosphere.

\section{References}

[1] Andersson, M., Passare, M., Sigurdsson, R.: Complex convexity and analytic functionals I. Preprint RH-06-95, Science Institute, University of Iceland, 1995

[2] Behnke, H., Peschl, E.: Zur Theorie der Funktionen mehrerer komplexer Veränderlichen. Konvexität in Bezug auf analytische Ebenen im Kleinen und Großen. Math. Ann. 111, 158-177 (1935)

[3] Berndtsson, B., Andersson, M.: Henkin-Ramirez formulas with weight factors. Ann. Inst. Fourier 32, 91-110 (1982)

[4] Boas, H.P., Straube, E.J.: On equality of type and variety type of real hypersurfaces in $\mathbb{C}^{n}$. J. Geom. Analysis 2, 95-98 (1992)

[5] Bruna, J., Nagel, A., Wainger, S.: Convex hypersurfaces and Fourier transform. Ann. Math. 127, 333-365 (1988)

[6] Diederich, K., Fischer, B., Fornæss, J.E.: Hölder estimates on convex domains of finite type. Math. Z. 232, 43-61 (1999)

[7] Diederich, K., Fornæss, J.E.: Support functions for convex domains of finite type. Math. Z. 230, 145-164 (1999)

[8] Diederich, K., Mazzilli, E.: Extension and restriction of holomorphic functions. Ann. Inst. Fourier 47, 1079-1099 (1997)

[9] Diederich, K., Mazzilli, E.: A remark on a theorem by Ohsawa-Takegoshi. Nagoya Math. J. 158, 185-189 (2000)

[10] Diederich, K., Mazzilli, E.: Extension of bounded holomorphic functions in convex domains. manuscripta math. 105, 1-12 (2001)

[11] Diederich, K., Mazzilli, E.: Zero varieties for the Nevanlinna class on all convex domains of finite type. Nagoya Math. J. 163, 215-227 (Preprint 1998) (2001)

[12] Fischer, Bert: $L^{p}$-estimates on convex domains of finite type. Math. Z. 236, 401-418 (2001)

[13] Fornæss, J.E.: Peak points on weakly pseudoconvex domains. Math. Ann. 227, 173175 (1977)

[14] Hefer, T.: Hölder and $L^{p}$-estimates for del-bar on convex domains of finite type depending on Catlin's multitype. Preprint, 2001 
[15] Henkin, G.M., Leiterer, J.: Andreotti-Grauert theory by integral formulas. Akademie-Verlag, Berlin, 1984

[16] Henkin, G.M., Leiterer, J.: Theory of Functions on Complex Manifolds. Monographs in Mathematics, vol. 79, Birkhäuser Verlag, Basel, 1984

[17] Kiselman, Chr.: Lineally convex Hartogs domains. Acta Math. Vietnamica 21, 69-94 (1996)

[18] Kiselman, Chr.: Duality of functions defined in lineally convex sets, Universitatis Iagellonicae. Acta Mathematica 35, 7-36 (1997)

[19] Kiselman, Chr.: A differential inequality characterizing weak lineal convexity. Math. Ann. 311, 1-10 (1998)

[20] Lieb, I., Michel, J.: The Cauchy-Riemann complex. Integral formulae and Neumann problem. Aspects of Mathematics, vol. E 34, Vieweg-Verlag, Wiesbaden, 2002

[21] Mazzilli, E.: Division et extension des fonctions holomorphes dans les ellipsoides, Ph.D. thesis, Université Paul Sabatier de Toulouse, 1995

[22] Mazzilli, E.: Extension des fonctions holomorphes. C. R. Acad. Sci. Paris 321, 837841 (1995)

[23] Mazzilli, E.: Extension des fonctions holomorphes dans les pseudo-ellipsoides. Math. Z. 227, 607-622 (1998) 